\title{
Synthesis of new dithia[3.3]parapara- and metapara-cyclophane based tectons: toward an universal surface-confined 2D/3D molecular binding motif
}

\author{
Elena Zaborova, ${ }^{\mathrm{a}, \mathrm{b}}$ Alice Six, ${ }^{\mathrm{a}}$ Hanane Amokrane, ${ }^{\mathrm{c}}$ Fabrice Charra, ${ }^{\mathrm{c}}$ Fabrice Mathevet, ${ }^{\mathrm{a}}$ David Kreher ${ }^{\mathrm{a}}{ }^{\mathrm{a}}$ and \\ André-Jean Attias ${ }^{\mathrm{a}^{*}}$ \\ a Sorbonne Universités, UPMC Univ Paris 06, CNRS, Institut Parisien de Chimie Moléculaire (IPCM), chimie \\ des polymères, 75005 Paris, France \\ ${ }^{b}$ Aix Marseille Universite, CNRS, CINaM UMR 7325, Campus de Luminy, Case 913, 13288 Marseille, France. \\ ' Service de Physique de l'Etat Condensé, SPEC CEA CNRS Université Paris-Saclay, CEA Saclay F-91191 Gif-sur- \\ Yvette CEDEX, France
}

\section{Introduction}

Long-range $2 \mathrm{D} / 3 \mathrm{D}$ self-assemblies of specific building blocks to form well defined architectures onto surfaces is an important challenge in Nanotechnology. ${ }^{1}$ To obtain these supramolecular structures, on one hand non-covalent interactions such as hydrogen bonding, ${ }^{2}$ van der Waals forces ${ }^{3}$ or metal-ligand, ${ }^{4}$ are commonly used. Moreover, usually the building blocks (tectons) are prepared to be able to form self-assemblies on one particular surface and the change of the substrate leads to the loss of the organization. On the other hand, the robustness under ambient conditions of the assembled monolayer is also an important issue to take into account in the design of new materials.

This can be achieved in taking advantage of physisorption strategies as for instance with the use of van der Waals forces promoting both physisorption of organic building blocks on the surfaces and strong lateral intermolecular interactions. In this context, stable monolayers could be reached on highly oriented pyrolytic graphite (HOPG) : ${ }^{5}$ in this approach, the robustness of the assembly depends on the judicious design of adsorbed tectons, presenting both an aromatic core and peripheral alkyl chains with a suitable distance between the alkyl chains to lead to their intermolecular interdigitation in the plane..

However, despite the fact that several applications require specific substrates as for example in molecular plasmonics requiring a noble metal substrate such as $\mathrm{Au}(111)$, a limited number of stable monolayers on $\mathrm{Au}(111)$ have been reported so far. ${ }^{6}$ Indeed, the challenge with the gold surface lays on the incommensurability between the linear hydrocarbon and the reconstructed $\mathrm{Au}(111)$ lattices. In this case, the formation of stable structures is much more sensitive to the number of the methylene groups per chain. As a consequence, the development of building blocks compatible with both substrates still remains challenging.

Moreover, in such fields like electronics and photonics, the extension of the two dimensional selfassembling materials to the third dimension is also an essential issue. Indeed, the close proximity of absorbed photoactive entities with conducting substrate leads to the quenching of any electronic excitations. To avoid this detrimental phenomenon, the active molecular units have to be decoupled from the conducting substrate. The raising of the molecules can be achieved by covering the surface with a uniform insulating layer ${ }^{7}$ or by introduction of the bulky groups like tert-butyl groups on the molecules. ${ }^{8}$ However, the main disadvantage of these two strategies is the limited size of in-plane domains. To solve this problem, supramolecular self-assemblies of 3D building blocks have shown to be an efficient approach allowing the regular assembly of the bottom part of 3D molecules by supramolecular interactions and forming the 2D layer directly in contact with the conducting surface. ${ }^{9}$ Thus, this kind of self-assemblies permits to control the positionning of the functional upper entities of the $3 \mathrm{D}$ tectons and to decouple them from the surface.

Based on this approach, we developed few years ago a functional "clip" (see Figure 1a), specifically designed for 2D supramolecular self-assembly confined on HOPG. ${ }^{10}$ This recognition motif consists of two pairs of alkyl chains bonded through a $\pi$-conjugated bistilbene-like bridge at twice the lateral distance between $n$-alkanes physisorbed on HOPG (8.52 $\AA$ ). This clip was designed in such a way that when two of these units interdigit on the surface, their self-assembly leads to the formation of lamellae that strictly pre- 
serve the Groszek geometry, ${ }^{11}$ which is one of the main driving forces of the self-assembly processes. This binding motif is able to steer the self-assembly of a large variety of tectons resulting in the formation of 2D supramolecular architectures of different topologies. Subsequently and more recently, to access to the third third dimension, we took advantage of the same strategy based on such van der Waals interactions to form $3 \mathrm{D}$ networks under $\mathrm{sp}^{2}$-carbone based substrates, including HOPG and graphene, starting from novel 3D tectons. These doubly-functionalized 3D tectons were designed to present two opposite faces liked by a cyclophane core; a lower face (pedestal) bearing the "clip" alkyl chains for guiding 2D self-assembly on the substrate (HOPG), and an upper face bearing a functional moiety. ${ }^{12}$ However, since it was specifically designed related to HOPG substrate, the latter clipping functional group doesn't allow to transfer this strategy to other conducting surfaces of interest such as $\mathrm{Au}(111)$ for example. .

This is why recently as well, we designed an "universal" alkyl chain-based surface-confined molecular binding motif, so-called "minimal clip" (see Figure 1b), in order to maximize 2D crystallization energies on different substrates. ${ }^{13}$ More precisely, we demonstrated its efficiency in designing the smallest bifunctional building block equipped with this minimal clip, self-assembling under ambient conditions in large monodomains consisting of closely packed non-covalent polymer-like structures with enhanced robustness, and steering molecular self-assemblies on both HOPG and Au(111) (Figures 1c and 1d).

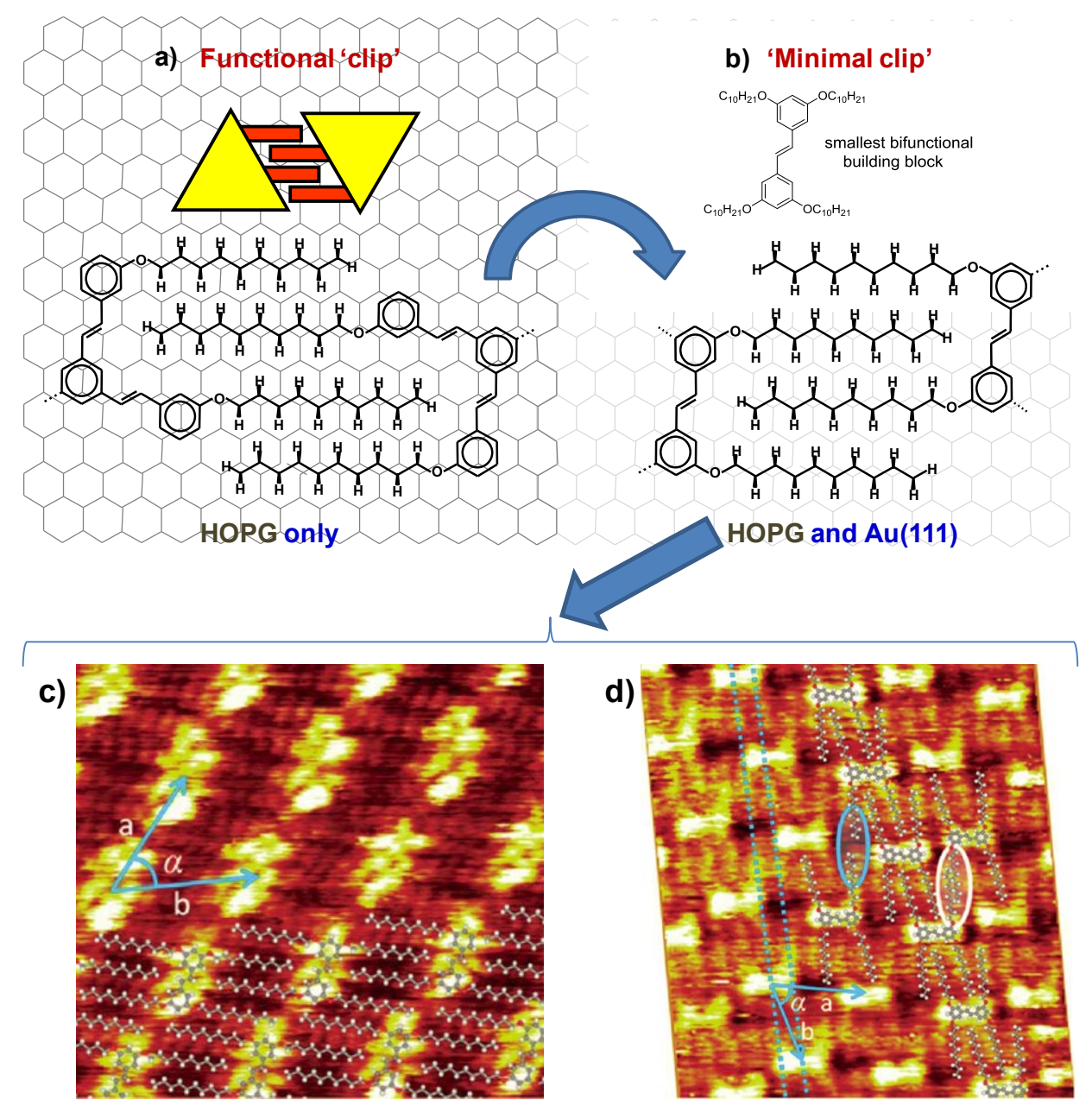

Figure 1. a) Molecular model of the "molecular clip" function on HOPG; b) Molecular model of the "minimal clip" function on HOPG; c) STM image of the self-assembly of the "minimal clip" molecule at the phenyloctane/HOPG interface and d) at the phenyloctane/Au(111) interface. (after ref. 13)

Nevertheless, the possibility to generalize and/or extend this "minimal clip" concept to other building blocks, and more particularly to 3D tectons, has never been considered so far, and the development of selfassembling three dimensional entities compatible with both HOPG and Au(111) surface are still needed. Moreover, the integration of cyclophane moities into the structure of this optimized "clip" is also synthetically challenging. 
In this context, we present here the design and synthesis of novel "universal" 3D tectons able to selfassemble on both HOPG and $\mathrm{Au}(111)$ surfaces, including a cyclophane core paving the way to access to the third dimension. More precisely, we designed two different families of tectons based on different molecular binding motifs. The first family is based on the "minimal clip" approach previously mentioned and consists of three tectons: a "model" 2D tecton $\mathbf{A}$ to study the self-assembly properties on surfaces of such building blocks, and two new 3D tectons (B and C) incorporating the "model" structure as lower face (pedestal), and dithia[3.3] paraparacyclophane and dithia[3.3]metaparacyclophane moieties as 3D building blocks (Figure 2).

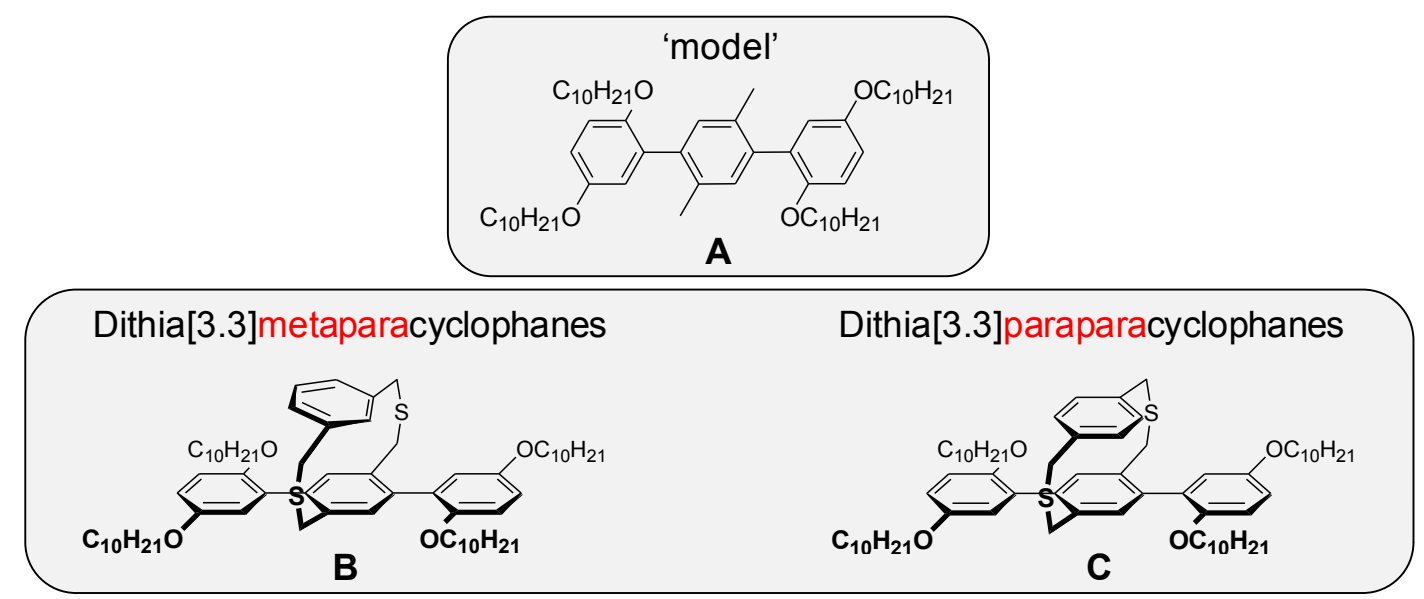

Figure 2. Chemical structures of the tecton family based on "minimal clip" approach (tectons A, B and C)

The second family is based on another binding motif approach. We replaced alkyl chains by carboxylic acid functions in order to test later-on the possibility to steer the self-assembly of such 3D tectons via hydrogen bonds. Indeed, this kind of interactions has been already used successfully for 2D self-assemblies on Au(111) as for example between terephthalic acid and terphenyl-3,3",5,5"'-tetracarboxylic acid derivatives(TPTC). ${ }^{14,15}$ Thus, this second family consist of five tectons: a "model" 2D diacid terphenyl tecton $D$ to study the selfassembly properties on surfaces of this kind of building blocks, and four new $3 D$ tectons $(\mathbf{E}, \mathbf{F}$ and $\mathbf{G}, \mathbf{H})$ incorporating diacid structures as lower face (pedestal) and dithia[3.3]paraparacyclophane and functionalized dithia[3.3]metaparacyclophane moieties as 3D building blocks (Figure 3). Preliminary encouraging scanning tunneling microscopy (STM) results will be presented as well.

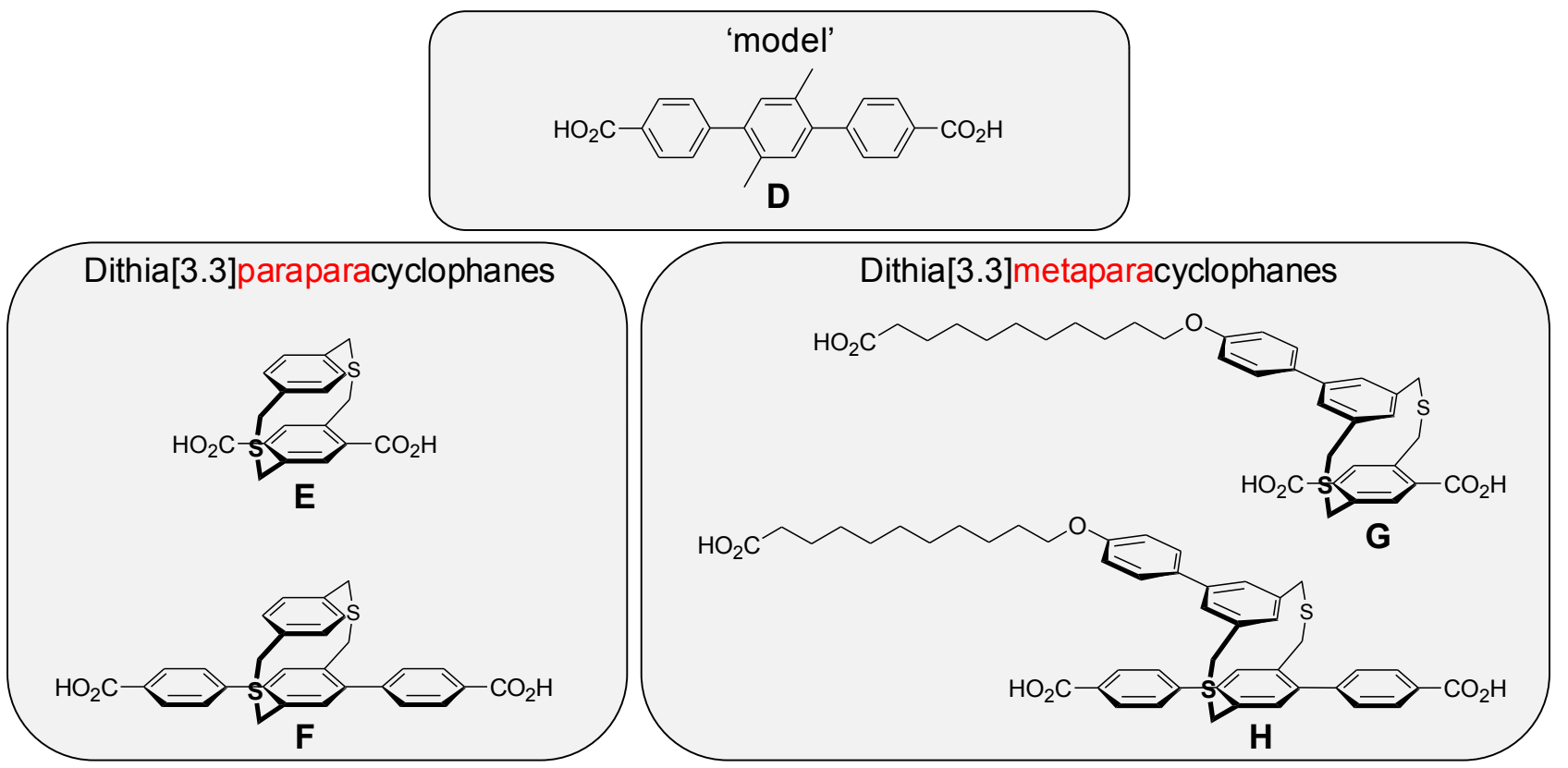

Figure 3. Chemical structures of the tecton family based on "hydrogen bonding" approach (tectons $\mathbf{D}, \mathbf{E}, \mathbf{F}$, $\mathbf{G}$ and $\mathbf{H})$ 


\section{Results and discussion}

\section{Synthesis of tectons A, B and C based on the "minimal clip" approach.}

Model 2D compound $\mathbf{A}$ was synthesized via a convergente synthetic strategy based on a direct Suzuki biscoupling between the 2,5-bis(decyloxy)phenyl boronic acid 3 and the 1,4-dibromo-2,5-dimethylbenzene core 4 in an excellent $77 \%$ yield (Scheme 2). Note that the choice of the dimethylbenzene 4 bearing two methyl groups have been done to mimic the presence of the methylene bridges in cyclophane moities, and that this convergente strategy also permits to easily replace the kind of central cores. Thus, the 3D tectons B was prepared following the same strategy in coupling two boronic derivatives $\mathbf{3}$ and the 1,4-dibromo-2,11dithia[3.3]metaparacyclophane 5 (under micro-wave conditions) with a yield of $43 \%$ (Scheme 1).

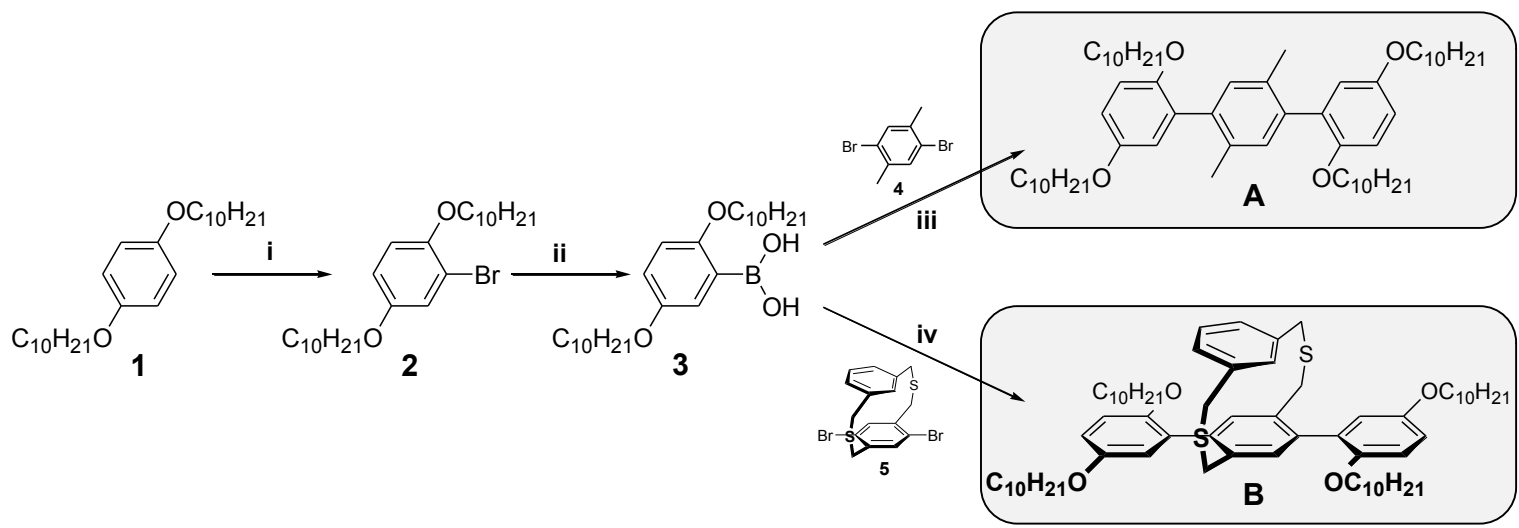

Scheme 1. Synthesis of the 2D model structure $\mathbf{A}$ and meta-paracyclophane based 3D tecton $\mathbf{B}$ with three phenyl rings and four dodecyloxy chains. i) $\mathrm{Br}_{2}, \mathrm{CCl}_{4}, 43 \%$; ii) Isopropoxyboronic acid pinacol ester, $n \mathrm{BuLi}$, THF, 72\%; iii) 4, $\mathrm{Pd}\left(\mathrm{PPh}_{3}\right)_{4}, \mathrm{~K}_{2} \mathrm{CO}_{3}, \mathrm{THF} / \mathrm{H}_{2} \mathrm{O}, 77 \%$; iv) 5, $\mathrm{Pd}\left(\mathrm{PPh}_{3}\right)_{4}, \mathrm{~K}_{2} \mathrm{CO}_{3}, \mathrm{THF} / \mathrm{H}_{2} \mathrm{O}, \mu$-waves, $43 \%$

Nevertheless, the synthesis of the 3D tecton C incorporating dithia[3.3]paraparacyclophane core didn't succeed via the previous convergente strategy, whatever under conventional heating or under micro-wave conditions. Indeed, the direct Suzuki coupling gives only mono-adduct. Consequently, another synthetic route was developed based on the formation of the cyclophane entity in a last cyclization step, as depicted in Scheme 2. More precisely, the 2,5-bis(decyloxy)phenyl boronic acid 3 was first bis-coupled with the 1,4dibromo-2,5-bis(methoxymethyl)benzene 6 to give the terphenyl intermediate 7 with a $95 \%$ yield. Then the alkoxy groups of the central phenyl core were selectively cleaved by addition of boron tribromide $\left(\mathrm{BBr}_{3}\right)$, to lead to the dibromomethyl compound $\mathbf{8}$ which was cyclized with (4-mercaptomethyl-phenyl)-methanethiol 9 to generate the 3D tecton $\mathbf{C}$ with a final $13 \%$ yield.
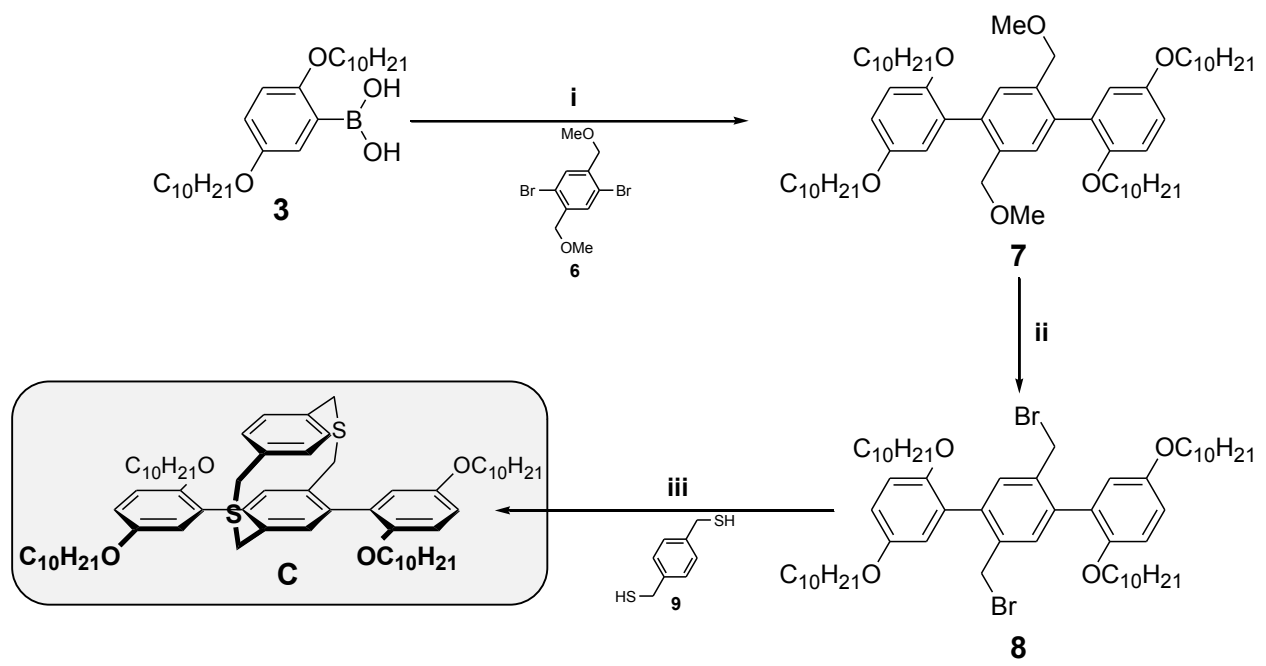

Scheme 2. Synthesis of 2,11-dithia[3.3] paracyclophane derivative C. i) $\mathrm{Pd}\left(\mathrm{PPh}_{3}\right)_{4}, \mathrm{~K}_{2} \mathrm{CO}_{3}, \mathrm{THF} / \mathrm{H}_{2} \mathrm{O}, 95 \%$; ii) $\mathrm{BBr}_{3}, \mathrm{DCM}, 42 \%$; iii) 9, $\mathrm{KOH}, \mathrm{DCM} / \mathrm{MeOH}, 13 \%$ 


\section{Synthesis of tectons D, E, F, G and H towards "hydrogen bonding" approach.}

The new tectons $\mathbf{D}, \mathbf{E}, \mathbf{F}, \mathbf{G}$ and $\mathbf{H}$ were designed in replacing the previous alkyl chain binding motifs by carboxylic acid functions in order to potentially take advantage of hydrogen bonding interactions to steer the self-assembly, whatever the substrate.

The first 3D tectons prepared based on this "hydrogen bonding" approach, are the compounds $\mathbf{E}$ and $\mathbf{G}$ which present the smallest pedestal that we can design (terephthalic acid as lower faces) and which bear a nude $(\mathbf{E})$ and functionalized $(\mathbf{G})$ upper faces. They were both prepared from diester compound 10, synthesized according to the literature ${ }^{16}$. Then, compound $\mathbf{1 0}$ underwent a cyclization with either dithiol $\mathbf{9}$ or $\mathbf{1 3}$, to give the paraparacyclophane 11 and the functionalized metaparacylophane 13, respectively. Finally, the tecton $\mathbf{E}$ was obtained by a simple hydrolysis of the intermediate 11, whereas the intermediate $\mathbf{1 3}$ was first functionalized with the boronic derivative $\mathbf{1 5}$ via a Suzuki coupling before hydrolysis to lead to the desired tecton $\mathbf{G}$ (Scheme 3).

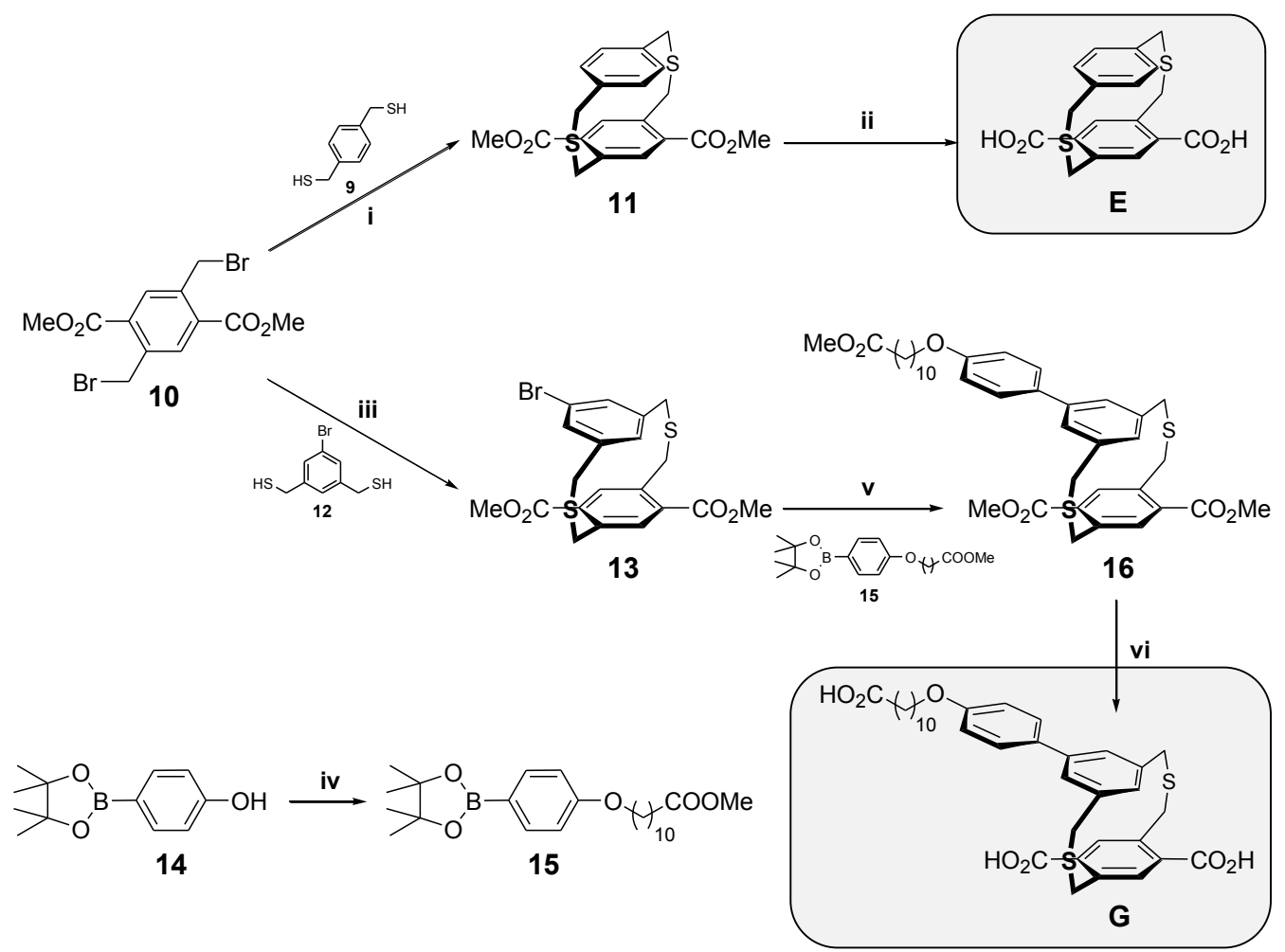

Scheme 3. Synthesis of 3D tectons E and G. i) $\mathrm{KOH}, \mathrm{DCM} / \mathrm{MeOH}, 55 \%$; ii) $\mathrm{KOH}, \mathrm{H}_{2} \mathrm{O} / \mathrm{THF}, 99 \%$; iii) $\mathrm{KOH}$, $\mathrm{DCM} / \mathrm{MeOH}, 59 \%$; iv) methyl 11-bromoundecanoate, $\mathrm{K}_{2} \mathrm{CO}_{3}, \mathrm{DMF}, 87 \%$; v) $\mathrm{Pd}\left(\mathrm{PPh}_{3}\right)_{4}, \mathrm{Na}_{2} \mathrm{CO}_{3}$, Toluene/EtOH/ $\mathrm{H}_{2} \mathrm{O}, 48 \%$; vi) $\mathrm{KOH}, \mathrm{H}_{2} \mathrm{O} / \mathrm{THF}, 70 \%$

The last tectons $\mathbf{D}, \mathbf{F}$ and $\mathbf{H}$ display a terphenyl pedestal. Model compound $\mathbf{D}$ has been prepared via a Suzuki bis-coupling between commercially available molecules 4 and 17, to first obtain the diester derivative 18, before undergoing a hydrolysis to reach to $\mathbf{D}$ in a quantitative yield (Scheme 4 ).

$>_{\mathrm{O}}^{\mathrm{O}}-\mathrm{CO}_{2} \mathrm{Me}$

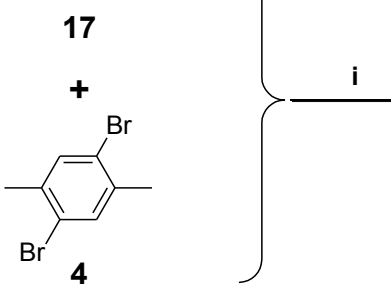

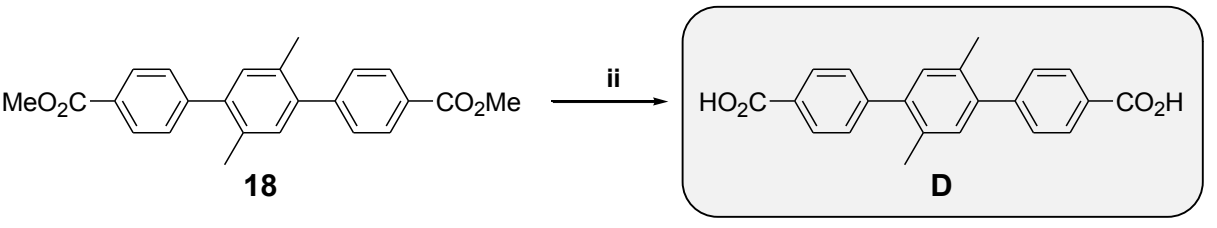

18

Scheme 4. Synthesis of "2D model" terphenyl D. i) $\mathrm{Pd}\left(\mathrm{PPh}_{3}\right)_{4}, \mathrm{~K}_{2} \mathrm{CO}_{3}, \mathrm{THF} / \mathrm{H}_{2} \mathrm{O}, 83 \%$; ii) $\mathrm{KOH}, \mathrm{H}_{2} \mathrm{O} / \mathrm{THF}, 99 \%$ 
The synthesis of both 3D tectons $\mathbf{F}$ and $\mathbf{H}$ is based on the key bis(brominated) terpheny intermediate $\mathbf{2 0}$ prepared via a Suzuki coupling reaction between $\mathbf{6}$ and 17, and following by a selective cleavage of methoxy groups with $\mathrm{BBr}_{3}$. Then, the key compound 20 reacted subsequently either with dithiol 6 to lead to the 3D "nude" tecton $\mathbf{F}$, or with dithiol $\mathbf{1 2}$ followed by a Suzuki coupling with compound $\mathbf{1 5}$ and then by a hydrolysis of the ester functions to lead to the functionalized tecton $\mathbf{H}$ (Scheme 5).
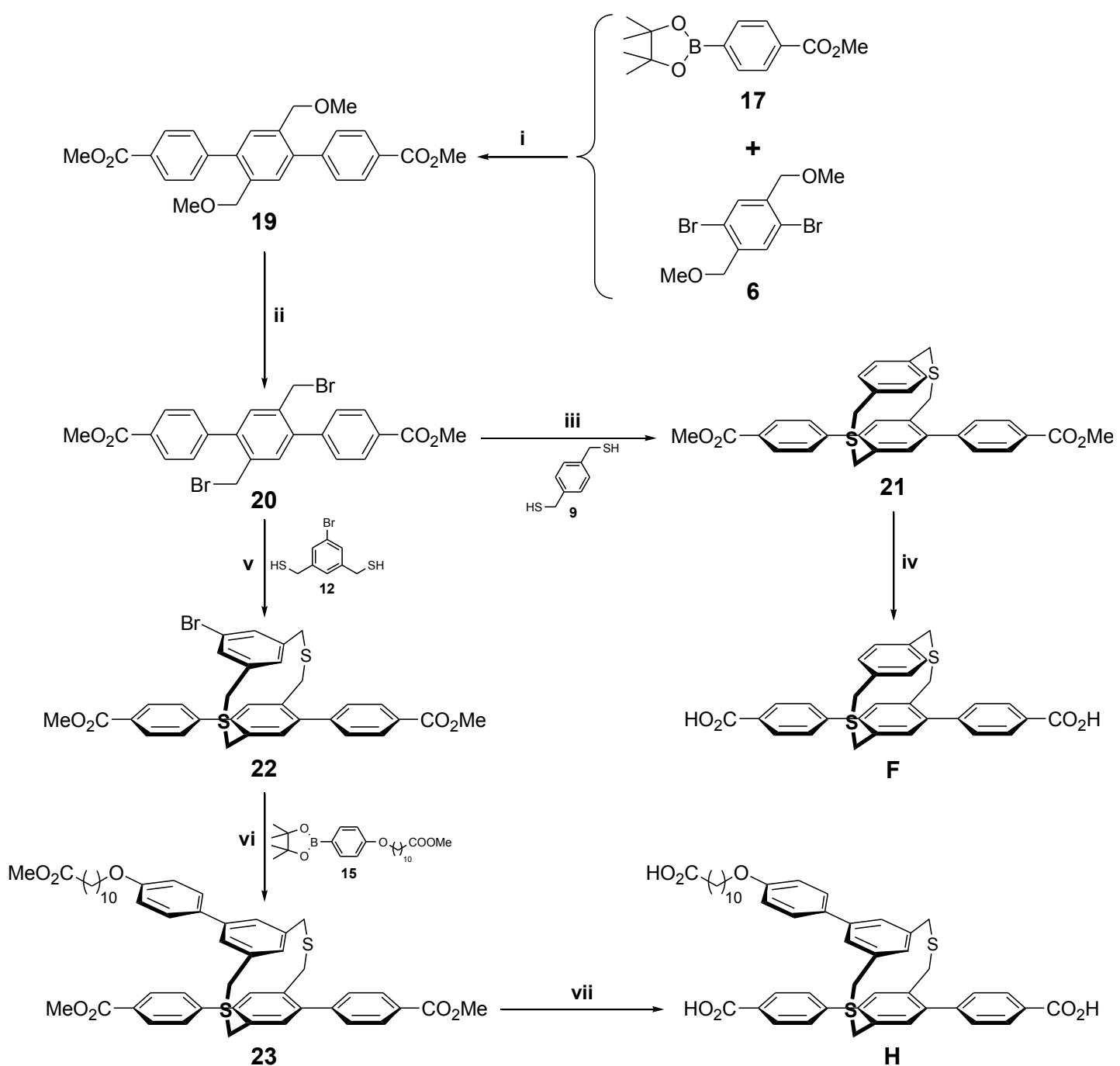

Scheme 5. Synthesis of terphenyl derivatives $\mathbf{F}$ and $\mathbf{H}$. i) $\mathrm{Pd}\left(\mathrm{PPh}_{3}\right)_{4}, \mathrm{~K}_{2} \mathrm{CO}_{3}, \mathrm{THF} / \mathrm{H}_{2} \mathrm{O}, 96 \%$; ii) $\mathrm{BBr}$, $\mathrm{DCM}, 99 \%$;

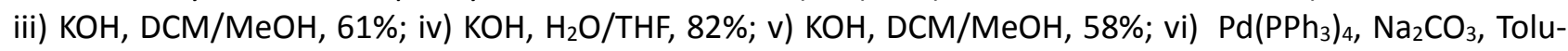
ene/EtOH/ $\mathrm{H}_{2} \mathrm{O}, 59 \%$; vii) $\mathrm{KOH}, \mathrm{H}_{2} \mathrm{O} / \mathrm{THF}, 95 \%$

All the intermediates and target tecton chemical structures were confirmed by usual techniques, i.e. NMR, chemical analysis and/or high resolution mass spectrometry, as reported in the experimental section.

\section{Preliminary self-assembly property study.}

This paper contribution deals essentially with the chemical strategies we developed to synthesize new cyclophane-based tectons, to investigate them later on as potential universal surface-confined 2D/3D molecular binding motifs.

Nevertheless, we already obtained preliminary results on the self-assembly properties of some of these tectons studied by STM at liquid-solid interface. For instance, all the compounds of the first family (A, B and C) self-assemble both on HOPG and Au(111), even if the stability of the self-assemblies (size of the domains) and the image resolutions are better on $\mathrm{Au}(111)$ substrate. Typical images of the self-assemblies obtained for tectons $\mathbf{A}$ and $\mathbf{C}$ on HOPG are reported in Figure $\mathbf{1} \mathbf{a}$ and $\mathbf{1} \mathbf{b}$ respectively, and typical images for the tecton $\mathbf{B}$ on $\mathrm{Au}(111)$ are presented in figures $\mathbf{1} \mathbf{c}-\mathbf{d}$. 
a)

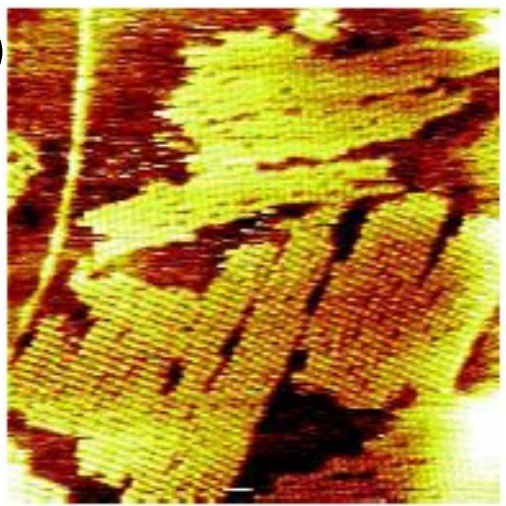

C)

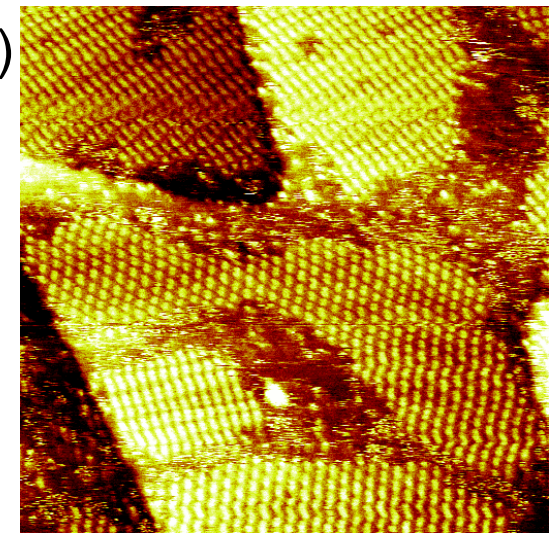

b)

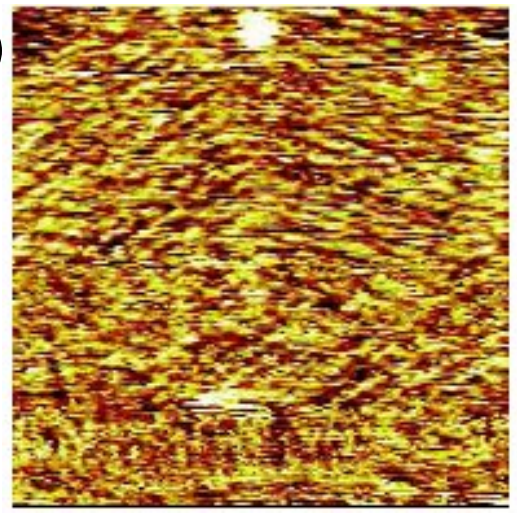

d)

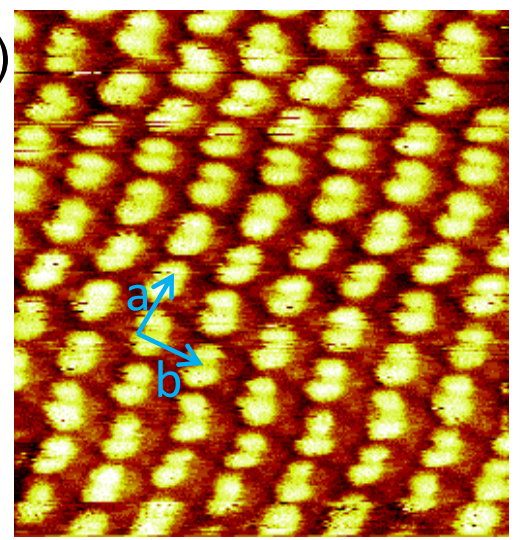

Figure 1. a) STM on HOPG at liquid-solid interface of tecton $\mathbf{A}, 123 * 123 \mathrm{~nm} 2$; b) STM on HOPG at liquidsolid interface of tecton $\mathbf{C}, \mathrm{I}_{\mathrm{T}}=15 \mathrm{pA} / \mathrm{V}_{\mathrm{T}}=800 \mathrm{mV} / 57^{*} 57 \mathrm{~nm}^{2} ; \mathbf{c}$ ) Long-distance STM image of tecton B under $\mathrm{Au}(111), \mathrm{I}_{\mathrm{T}}=3 \mathrm{pA} / \mathrm{V}_{\mathrm{T}}=-300 \mathrm{mV} / 100 \times 100 \mathrm{~nm}^{2} ; \mathrm{d}$ ) Zoom-in of tecton $\mathbf{B}$ on Au with constant current mode, $\mathrm{l}_{\mathrm{T}}=6 \mathrm{pA} / \mathrm{V}_{\mathrm{T}}=-840 \mathrm{mV} / 19 \times 19 \mathrm{~nm}^{2}$.

As expected from the design, all the target tectons A-C self-assemble onto HOPG thanks to the long peripheral alkoxy chains. The planar 2D model tecton A (Fig. 1a) form relatively small domains, estimated about few tens of nanometers squared. The structures observed evidence well-ordered supramolecular linear polymers type topologies with different orientations. Nevertheless, the resolution is not high enough to determine the precise organization into each non-covalent polymer-like chain, even at small scale.

More importantly, these preliminary results evidence that the 3D tectons based on the "minimal clip" approach and bearing a cyclophane as central core (metapara or parapara) give self-assemblies on $\mathrm{Au}(111)$ as well, at the liquid solid interface (Figure 1c). Moreover, such images are for the moment recorded easily compared to HOPG substrate and better-ordered self-assemblies are obtained, under larger scale domains: based on small scale resolution, it was even possible to determine the lattice parameters for $\mathbf{B}$, which are: a $=1,9 \mathrm{~nm} ; \mathrm{b}=2,4$; Angle $=97^{\circ}$ (Fig. 1d).

Further STM investigations with tectons D, E, F, G and $\mathrm{H}$ are also currently in progress to try to determine the respective practical circumstances in which either "minimal clip" or "hydrogen bonding" approaches is the most compliant surface-confined 2D/3D molecular binding motif.

\section{Conclusions}

A series of novel "universal" 2D and 3D tectons including parapara-, metapara- or functionalized metaparacyclophanes has been designed for supramolecular self-assembly on both HOPG and Au surfaces. These tectons were synthesized in incorporating two different molecular binding motifs based on either a "minimal clip" or a "hydrogen bonding" approach. The versatility of the synthetic routes paves the way to more complex 3D tectons bearing a large variety of functional units such as chromophores or photoswitchable molecules.... Moreover, preliminary STM investigations show that these compounds successfully selfassemble both on HOPG and $\mathrm{Au}(111)$ substrates at liquid-solid interface. Thus, these promising results on the elaboration of potential decoupled functional 3D self-assemblies on plasmonic substrates such as $\mathrm{Au}(111)$ open-up great opportunities in the field of Nanoscience. 


\section{Experimental section}

\section{Chemistry}

Tetrahydrofurane (THF), Toluene and Dichloromethane $\left(\mathrm{CH}_{2} \mathrm{Cl}_{2}\right)$ used in synthesis come from a MBRAUN fountain model MB SPS-800. All other solvents were purchased from commercial suppliers and used without further purification. Chromatographic separations were carried out with a flash chromatographic instrument (Grace, Reveleris) and with silica gel (40 $\mathrm{m}$, Grace). ${ }^{1} \mathrm{H}$ NMR spectra were recorded at $200 \mathrm{MHz}$, proton chemical shifts $(\delta)$ are reported in ppm and referenced to the residual solvent signal: $\mathrm{CDCl}_{3}(7.26)$, MeOD (3.31) and DMSO-d 6 (2.50). ${ }^{13} \mathrm{C}$ NMR spectra were recorded at $200 \mathrm{MHz}$ and $\delta$ referenced to the residual solvent signal: $\mathrm{CDCl}_{3}$ (77.16), $\mathrm{MeOD}(49.0)$ and $\mathrm{DMSO}-\mathrm{d}_{6}$ (39.52).

\section{1,4-Didecyloxybenzene (1).}

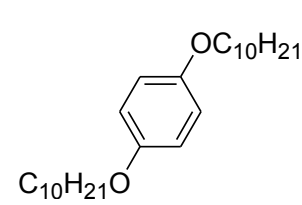

A solution of hydroquinone $(5 \mathrm{~g}, 0.045 \mathrm{~mol}), 1$-bromodecane $(30.06 \mathrm{~g}, 0.136 \mathrm{~mol})$ and $\mathrm{K}_{2} \mathrm{CO}_{3}(25.1 \mathrm{~g}, 0.182 \mathrm{~mol})$ into butanone $(100 \mathrm{~mL})$ was heated under reflux for 2 days, and then filtered hot, with several washings with hot toluene. After removing the solvent under vacuum, the crude mixture was recrystallized with ethanol, leading after 1 night into the fridge to a white solid which was filtered and washed with cold ethanol.

Yield: $15 \mathrm{~g}(85 \%) . \%) .{ }^{1} \mathrm{H}$ NMR $\left(\mathrm{CDCl}_{3}, 200 \mathrm{MHz}\right): \delta 6.81$ (d, 4H), 3.95 (t, $4 \mathrm{H}, J=6.4 \mathrm{~Hz}$ ), 1.76 (quint, $4 \mathrm{H}$, $J=6.5 \mathrm{~Hz}), 1.53-1.22(\mathrm{~m}, 28 \mathrm{H}), 0.88(\mathrm{t}, 6 \mathrm{H}, J=6.4 \mathrm{~Hz}) .{ }^{13} \mathrm{C} \mathrm{NMR}\left(\mathrm{CDCl}_{3}, 50 \mathrm{MHz}\right): \delta 149.7,114.8,71.2,31.9$, $29.6,29.5,29.3,29.26,29.25,26.0,22.7,14.1$.

\section{1-Bromo-2,5-bis(decyloxy)benzene (2). ${ }^{17,18}$}

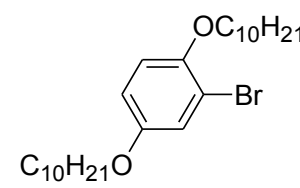

Solution of bromine $(2.6 \mathrm{~mL}, 51.20 \mathrm{mmol})$ in tetrachloromethane $(40.0 \mathrm{~mL})$ was added dropwise to a stirred solution of 1,4-didecyloxybenzene $1(20.0 \mathrm{~g}, 51.20 \mathrm{mmol})$ in tetrachloromethane $(60.0 \mathrm{~mL})$ under argon. The mixture was heated under reflux overnight, cooled, and then washed with water, saturated aqueous sodium metabisulfite, and water again, and the organic layer was dried $\left(\mathrm{MgSO}_{4}\right)$, and filtered. The solvent was removed in vacuo and the crude product was purified by column chromatography (silica gel/ PE with gradual introduction of $\mathrm{CH}_{2} \mathrm{Cl}_{2}$ ) and recrystallized from ethanol to give a white solid.

Yield: $10.3 \mathrm{~g}(43 \%) .{ }^{1} \mathrm{H}$ NMR $\left(\mathrm{CDCl}_{3}, 200 \mathrm{MHz}\right): \delta 7.11(\mathrm{~s}, 1 \mathrm{H}), 6.83-6.77(\mathrm{~m}, 2 \mathrm{H}), 3.95(\mathrm{t}, 2 \mathrm{H}, J=6.4 \mathrm{~Hz})$, $3.88\left(\mathrm{t}, 2 \mathrm{H}, J=6.4 \mathrm{~Hz}\right.$ ), 1.76 (quint, $4 \mathrm{H}, J=6.5 \mathrm{~Hz}$ ), $1.53-1.22(\mathrm{~m}, 28 \mathrm{H}), 0.88\left(\mathrm{t}, 6 \mathrm{H}, J=6.4 \mathrm{~Hz}\right.$ ). ${ }^{13} \mathrm{C} \mathrm{NMR}$ ( $\left.\mathrm{CDCl}_{3}, 50 \mathrm{MHz}\right): \delta 153.6,149.7,119.5,114.7,114.4,112.8,70.2,68.8,31.9,29.6,29.5,29.3,29.26,29.25$, 26.0, 22.7, 14.1 .

\section{2,5-Bis(didecyloxy)phenylboronic acid (3).}

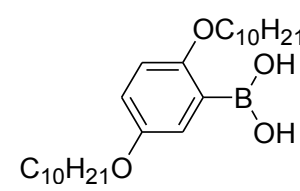

A solution of $n$-butillithium $(3.65 \mathrm{~mL}, 9.14 \mathrm{mmol}$ ) was added dropwise to a stirred, cooled $\left(-78^{\circ} \mathrm{C}\right)$ solution of 1-bromo-2,5-didecyloxybenzene $(1.95 \mathrm{~g}, 4.15 \mathrm{mmol})$ in dry THF $(75 \mathrm{~mL})$ under an argon atmosphere. The reaction mixture was stirred at $-78^{\circ} \mathrm{C}$ for $1 \mathrm{~h}$, then 2-isopropoxy-4,4,5,5-tetramethyl-1,3,2-dioxaborolane (1.86 mL, $9.14 \mathrm{mmoL})$ was added dropwise, and the mixture was allowed to attain room temperature overnight. Water $(50 \mathrm{~mL})$ was added and the product was extracted into ether. The combined ether layers were washed with water, dried $\left(\mathrm{MgSO}_{4}\right)$, and filtered. The solvent was removed in vacuo and the crude product was purified by column chromatography $\left(\mathrm{CH}_{2} \mathrm{Cl}_{2} / \mathrm{EtOAC}\right)$ to give compound $\mathbf{3}$ as a white solid.

Yield: $1.29 \mathrm{~g}(72 \%) .{ }^{1} \mathrm{H}$ NMR (THF, $\left.200 \mathrm{MHz}\right): \delta 7.54-7.49(\mathrm{~m}, 1 \mathrm{H}), 7.3(\mathrm{~s}, 2 \mathrm{H}), 7.58-7.09(\mathrm{~m}, 2 \mathrm{H}), 4.19(\mathrm{t}$, $2 \mathrm{H}, J=6.5 \mathrm{~Hz}), 4.07(\mathrm{t}, 2 \mathrm{H}, J=6.5 \mathrm{~Hz}), 2.05-1.82(\mathrm{~m}, 4 \mathrm{H}), 1.73-1.38(\mathrm{~m}, 28 \mathrm{H}), 1.07(\mathrm{t}, 6 \mathrm{H}, J=6.4 \mathrm{~Hz})$.

\section{1,4-Dibromo-2,5-dimethylbenzene (4). Commercially available.}

\section{Compound A.}

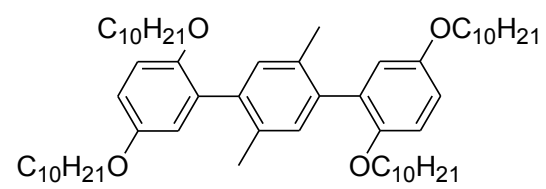

Degassed THF $(3.0 \mathrm{~mL})$ and water $(1.5 \mathrm{~mL})$ were added to a stirred, degassed mixture of 2,5-bis(decyloxy)phenyl boronic acid 3 (300 mg, $0.690 \mathrm{mmol}), 1,4$-dibromo-2,5-dimethylbenzene 4 (91 $\mathrm{mg}, 0.345 \mathrm{mmol}$ ), potassium carbonate $(382 \mathrm{mg}, 2.762 \mathrm{mmol})$ and tetrakis(triphenylphosphine)palladium(0) $(40 \mathrm{mg}, 0.0345 \mathrm{mmol}$ ). The 
mixture was heated under reflux for $48 \mathrm{~h}$ and cooled. Water was added, and the crude product was extracted into $\mathrm{CH}_{2} \mathrm{Cl}_{2}$. The combined $\mathrm{CH}_{2} \mathrm{Cl}_{2}$ extracts were washed with brine and dried $\left(\mathrm{MgSO}_{4}\right)$. The desiccant was filtered off, the solvent was removed in vacuo, and the crude product was purified by column chromatography $\left(\mathrm{PE} / \mathrm{CH}_{2} \mathrm{Cl}_{2}\right)$ to give compound $\mathbf{A}$ as a white solid.

Yield: $236 \mathrm{mg}$ (77\%). $R_{f} 0.25$ (PE/CH2Cl2: 8/2). mp: $86^{\circ} \mathrm{C} .{ }^{1} \mathrm{H}$ NMR $\left(\mathrm{CDCl}_{3}, 200 \mathrm{MHz}\right): \delta 7.07$ (s, 2H), $6.94-$ $6.78(\mathrm{~m}, 6 \mathrm{H}), 3.94(\mathrm{t}, 4 \mathrm{H}, J=6.5 \mathrm{~Hz}), 3.83(\mathrm{t}, 4 \mathrm{H}, J=6.5 \mathrm{~Hz}), 2.16(\mathrm{~s}, 6 \mathrm{H}), 1.84-1.71(\mathrm{~m}, 4 \mathrm{H}), 1.71-1.14(\mathrm{~m}$, $60 \mathrm{H}), 0.98-0.82(\mathrm{~m}, 12 \mathrm{H}) .{ }^{13} \mathrm{C} \mathrm{NMR}\left(\mathrm{CDCl}_{3}, 50 \mathrm{MHz}\right): \delta 153.2,150.6,137.5,133.4,132.9,131.3,117.6$, $114.5,113.9,69.7,68.7,32.1,29.9-29.4,26.3,26.1,22.84,22.83,19.7,14.3,14.2$. HRMS (ESI): calcd. for $\mathrm{C}_{60} \mathrm{H}_{98} \mathrm{O}_{4} \mathrm{Na}[\mathrm{M}+\mathrm{Na}]^{+}:$905.7357; found: 905.7396 (-4.3 ppm).

$5^{2}, 5^{5}$-Dibromo-3,7-dithia-1(1,3)-benzena-5(1,4)-benzenacyclooctaphane (5).

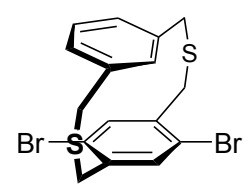

A solution (160 mL) of (2,5-dibromo-1,4-phenylene)dimethanethiol $(1.0 \mathrm{~g}, 3.05 \mathrm{mmol})$ and 1,3-bis-bromomehtyl-benzene ( $805 \mathrm{mg}, 3.05 \mathrm{mmol}$ ) in $\mathrm{CH}_{2} \mathrm{Cl}_{2}$ was added drop wise to a stirred methanol solution $(240 \mathrm{~mL})$ of $\mathrm{KOH}(214 \mathrm{mg}, 3.81 \mathrm{mmol})$ during $8 \mathrm{~h}$. After addition of a half volume of the $\mathrm{CH}_{2} \mathrm{Cl}_{2}$ solution a second portion of $\mathrm{KOH}(214 \mathrm{mg}, 3.81 \mathrm{mmol})$ was added to the methanol solution. The solvents were evaporated, water $(150 \mathrm{~mL})$ and $\mathrm{CH}_{2} \mathrm{Cl}_{2}$ $(150 \mathrm{~mL})$ were added, and the product was extracted into $\mathrm{CH}_{2} \mathrm{Cl}_{2}$, the combined $\mathrm{CH}_{2} \mathrm{Cl}_{2}$ extracts were dried over $\mathrm{MgSO}_{4}$, filtered, and concentrated under vacuum. The crude product was purified by column chromatography $\left(\mathrm{PE} / \mathrm{CH}_{2} \mathrm{Cl}_{2}\right)$ and recrystallized from toluene to give compound $\mathbf{5}$ as a white solid.

Yield: $782 \mathrm{mg}(60 \%) .{ }^{1} \mathrm{H}$ NMR $\left(\mathrm{CDCl}_{3}, 200 \mathrm{MHz}\right): \delta 7.38-7.02(\mathrm{~m}, 5 \mathrm{H}), 5.88(\mathrm{~s}, 1 \mathrm{H}), 4.15(\mathrm{~d}, 2 \mathrm{H}, J=13.3 \mathrm{~Hz})$, $3.71(\mathrm{~d}, 2 \mathrm{H}, J=13.3 \mathrm{~Hz}), 3.60(\mathrm{~d}, 2 \mathrm{H}, J=15.4 \mathrm{~Hz}), 3.50(\mathrm{~d}, 2 \mathrm{H}, J=15.4 \mathrm{~Hz}) ;{ }^{13} \mathrm{C} \mathrm{NMR}\left(\mathrm{CDCl}_{3}, 75 \mathrm{MHz}\right): \delta 139.1$, $138.2,135.0,128.4,127.4,126.0,123.1,35.8,34.8$.

\section{Compound B.}

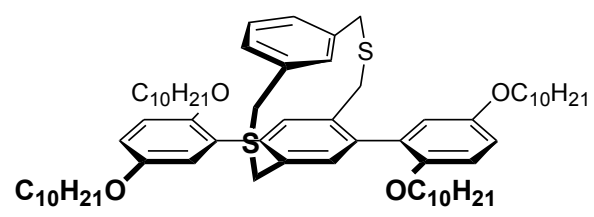

In a flame-dried micro-wave tube reactor equipped with a magnetic stirrer containing 2,5-bis(decyloxy)phenyl boronic acid 3 (130 mg, $0.299 \mathrm{mmol})$, compound 5 (64 $\mathrm{mg}, 0.150 \mathrm{mmol}$ ), potassium carbonate (165 mg, $1.196 \quad \mathrm{mmol})$ and tetrakis(triphenylphosphine)palladium(0) $(17 \mathrm{mg}, 0.015 \mathrm{mmol}$ ) under argon, were introduced degassed THF $(1.2 \mathrm{~mL})$ and degassed water $(0.6 \mathrm{~mL})$. The reaction mixture was heated with micro-waves at $150^{\circ} \mathrm{C}$ for $1.5 \mathrm{~h}$. and allowed to cool. Water $(4 \mathrm{~mL})$ and $\mathrm{CH}_{2} \mathrm{Cl}_{2}(4 \mathrm{~mL})$ were added, and the product was extracted into $\mathrm{CH}_{2} \mathrm{Cl}_{2}$; the combined $\mathrm{CH}_{2} \mathrm{Cl}_{2}$ extracts were dried over $\mathrm{MgSO}_{4}$, filtered, and concentrated under vacuum. The crude product was purified by column chromatography (PE/ $\mathrm{CH}_{2} \mathrm{Cl}_{2}$ 9/1) to yield compound $\mathbf{B}$ as a colorless oil.

Yield: $67 \mathrm{mg}$ (43\%). $R_{f} 0.63$ (PE/ $\left.\mathrm{CH}_{2} \mathrm{Cl}_{2}: 1 / 1\right) ;{ }^{1} \mathrm{H} \mathrm{NMR}\left(\mathrm{C}_{2} \mathrm{D}_{2} \mathrm{Cl}_{4}, 500 \mathrm{MHz}\right): \delta 7.09-7.02(\mathrm{~m}, 3 \mathrm{H}), 6.82(\mathrm{dd}, 2 \mathrm{H}$, $J=7.6 \mathrm{~Hz}), 6.78-6.72(\mathrm{~m}, 4 \mathrm{H}), 6.26(\mathrm{bs}, 2 \mathrm{H}), 6.12(\mathrm{bs}, 1 \mathrm{H}), 3.88(\mathrm{~d}, 2 \mathrm{H}, J=13.6 \mathrm{~Hz}), 3.85(\mathrm{t}, 4 \mathrm{H}, J=6.6 \mathrm{~Hz})$, $3.81-3.69(\mathrm{~m}, 2 \mathrm{H}), 3.68-3.59(\mathrm{~m}, 2 \mathrm{H}), 3.63(\mathrm{~d}, 2 \mathrm{H}, J=13.6 \mathrm{~Hz}), 3.47(\mathrm{~d}, 2 \mathrm{H}, J=15.0 \mathrm{~Hz}), 3.38(\mathrm{~d}, 2 \mathrm{H}$, $J=15.0 \mathrm{~Hz}), 1.74-1.67(\mathrm{~m}, 4 \mathrm{H}), 1.49-1.37(\mathrm{~m}, 8 \mathrm{H}), 1.37-1.07(\mathrm{~m}, 52 \mathrm{H}), 0.83-0.75(\mathrm{~m}, 12 \mathrm{H}) ;{ }^{13} \mathrm{C} \mathrm{NMR}$ $\left(\mathrm{C}_{2} \mathrm{D}_{2} \mathrm{Cl}_{4}, 125 \mathrm{MHz}\right): \delta 153.8,150.5,141.0,138.0,137.1,132.7,131.7,128.5,127.8,127.3,119.4,115.5$, $114.9,100.1,37.0,36.0,32.2,32.1,30.3-29.2,26.5,26.3,22.9,22.8,14.3$; HRMS (ESI): calcd. for $\mathrm{C}_{68} \mathrm{H}_{104} \mathrm{O}_{4} \mathrm{~S}_{2} \mathrm{Na}[\mathrm{M}+\mathrm{Na}]^{+}:$1071.7268; found: 1071.7266 (+0.2 ppm).

1,4-Dibromo-2,5-bis(methoxymethyl)benzene (6). Following the procedure of J-N. Moorthy et al. ${ }^{19}$

\section{2',5'-Bis(methoxymethyl)-2,2', 5,5''-tetradecyloxy-1,1':4',1'-terphenyl (7).}

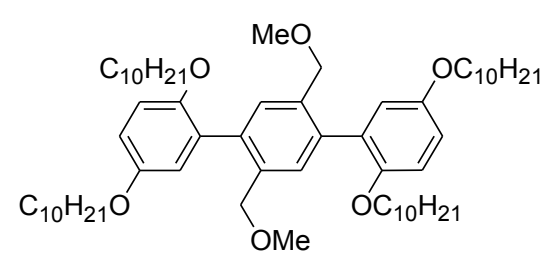

Degassed THF $(3.0 \mathrm{~mL})$ and water $(1.5 \mathrm{~mL})$ were added to a stirred, degassed mixture of 2,5-bis(decyloxy)phenyl boronic acid $3(300 \mathrm{mg}$, $0.690 \mathrm{mmol}$ ), 1,4-dibromo-2,5-bis(methoxymethyl)benzene 6 (112 mg, $0.345 \mathrm{mmol})$, potassium carbonate $(381 \mathrm{mg}, 2.762 \mathrm{mmol})$ and tetrakis(triphenylphosphine) palladium(0) $(40 \mathrm{mg}, 0.0345 \mathrm{mmol})$. The mixture was heated under reflux for $24 \mathrm{~h}$ and cooled. Water was added, and the crude product was extracted into $\mathrm{CH}_{2} \mathrm{Cl}_{2}$. The combined $\mathrm{CH}_{2} \mathrm{Cl}_{2}$ extracts were washed with brine and 
dried $\left(\mathrm{MgSO}_{4}\right)$. The desiccant was filtered off, the solvent was removed in vacuo, and the crude product was purified by column chromatography $\left(\mathrm{PE} / \mathrm{CH}_{2} \mathrm{Cl}_{2}\right)$ to give the product as a white solid.

Yield: $310 \mathrm{mg}$ (95\%). $R_{f} 0.39$ (PE/ $\left.\mathrm{CH}_{2} \mathrm{Cl}_{2}: 1 / 1\right) . \mathrm{mp}: 69^{\circ} \mathrm{C} .{ }^{1} \mathrm{H} \mathrm{NMR}\left(\mathrm{CDCl}_{3}, 200 \mathrm{MHz}\right): \delta 7.38(\mathrm{~s}, 2 \mathrm{H}), 6.86(\mathrm{~s}$, $6 \mathrm{H}), 4.34(\mathrm{bd}, 4 \mathrm{H}, J=14.7 \mathrm{~Hz}), 3.93(\mathrm{t}, 4 \mathrm{H}, J=6.5 \mathrm{~Hz}), 3.79(\mathrm{t}, 4 \mathrm{H}, J=6.3 \mathrm{~Hz}), 3.24(\mathrm{~s}, 6 \mathrm{H}), 1.86-1.68(\mathrm{~m}, 4 \mathrm{H})$, $1.61-1.14(\mathrm{~m}, 60 \mathrm{H}), 0.99-0.79(\mathrm{~m}, 12 \mathrm{H}) .{ }^{13} \mathrm{C} \mathrm{NMR}\left(\mathrm{CDCl}_{3}, 50 \mathrm{MHz}\right): \delta 153.2,150.5,136.8,135.4,131.5$, 129.3, 117.7, 114.4, 114.3, 72.3, 69.7, 68.8, 58.1, 32.1, 29.8 - 29.4, 26.2, 26.0, 22.83, 22.77, 14.27, 14.25. HRMS (ESI): calcd. for $\mathrm{C}_{62} \mathrm{H}_{102} \mathrm{O}_{6} \mathrm{Na}[\mathrm{M}+\mathrm{Na}]^{+}:$965.7569; found: 965.7572 (-0.3 ppm). calcd. for $\mathrm{C}_{62} \mathrm{H}_{102} \mathrm{O}_{6} \mathrm{~K}$ $[\mathrm{M}+\mathrm{K}]^{+}$: 981.7308; found: 981.7298 (+1.0 ppm).

\section{2',5'-Bis(bromomethyl)-2,2',5,5'-tetradecyloxy-1,1':4',1'-terphenyl (8).}

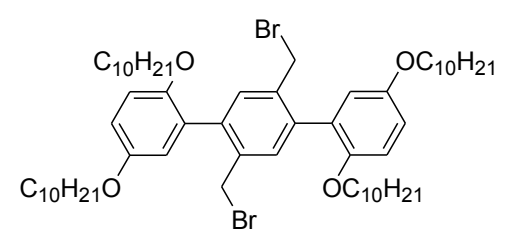

To the dry $\mathrm{CH}_{2} \mathrm{Cl}_{2}$ solution $(3.0 \mathrm{~mL})$ of $7\left(150 \mathrm{mg}, 0.159^{\circ} \mathrm{mmol}\right)$ was added boron tribromide (1.0 M in $\mathrm{CH}_{2} \mathrm{Cl}_{2}, 0.36 \mathrm{~mL}$ ) under an argon atmosphere. The reaction mixture was stirred at room temperature under argon for $2^{\circ} \mathrm{h}$, then quenched with water at $0^{\circ} \mathrm{C}$, and extracted with $\mathrm{CH}_{2} \mathrm{Cl}_{2}$. The organic layer was washed with brine, dried over $\mathrm{MgSO}_{4}$, filtered, and evaporated. The crude product was purified by column chromatography $\left(\mathrm{PE} / \mathrm{CH}_{2} \mathrm{Cl}_{2}\right)$ to give the product 8 as a white solid.

Yield: $69 \mathrm{mg}(42 \%) \cdot R_{f} 0.50$ (PE/ $\left.\mathrm{CH}_{2} \mathrm{Cl}_{2}: 3 / 1\right) \cdot \mathrm{mp}: 85^{\circ} \mathrm{C} .{ }^{1} \mathrm{H}$ NMR $\left(\mathrm{CDCl}_{3}, 200 \mathrm{MHz}\right): \delta 7.38(\mathrm{~s}, 2 \mathrm{H}), 6.95-6.87$ $(\mathrm{m}, 6 \mathrm{H}), 4.41(\mathrm{bd}, 4 \mathrm{H}, J=4.5 \mathrm{~Hz}), 3.96(\mathrm{t}, 4 \mathrm{H}, J=6.6 \mathrm{~Hz}), 3.82(\mathrm{bdd}, 4 \mathrm{H}, J=5.6 \mathrm{~Hz}, J=6.6 \mathrm{~Hz}), 1.96-1.68(\mathrm{~m}$, $4 \mathrm{H}), 1.52-1.13(\mathrm{~m}, 60 \mathrm{H}), 0.96^{\circ}-0.79(\mathrm{~m}, 12 \mathrm{H}) .{ }^{13} \mathrm{C} \mathrm{NMR}\left(\mathrm{CDCl}_{3}, 50 \mathrm{MHz}\right): \delta 153.3,150.2,137.9,136.0$, 132.8, 129.8, 117.3, 115.3, 114.4, 69.7, 68.8, 32.0, 29.9-28.8, 26.2, 26.0, 22.8, 14.3, 14.2. HRMS (ESI): calcd. for $\mathrm{C}_{60} \mathrm{H}_{96} \mathrm{Br}_{2} \mathrm{O}_{4} \mathrm{Na}[\mathrm{M}+\mathrm{Na}]^{+}$: 1061.5568; found: 1061.5548 (+1.8 ppm). calcd. for $\mathrm{C}_{60} \mathrm{H}_{96} \mathrm{Br}_{2} \mathrm{O}_{4} \mathrm{~K}[\mathrm{M}+\mathrm{K}]^{+}$: 1077.5307; found: 1077.5267 (+3.7 ppm).

\section{1,4-Dimercaptomethylbenzene (9). ${ }^{20}$}

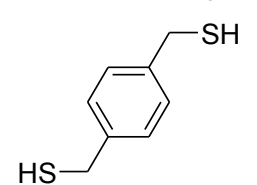

First, in a $50 \mathrm{ml}$ flask, 1,4-dibromomethyl-benzene $(2.0 \mathrm{~g}, 7.577 \mathrm{mmol})$ was introduced, before adding successively thiourea $(1.21 \mathrm{~g}, 15.91 \mathrm{mmol})$ and ethanol $(15 \mathrm{~mL})$ : after $4 \mathrm{~h}$ at reflux and cooling to room temperature, the solvent was evaporated under vacuum. Second, $\mathrm{KOH}(2.13 \mathrm{~g}, 37.89 \mathrm{mmol})$ and water $(15 \mathrm{ml})$ were added and the mixture was heated at reflux for $6 \mathrm{~h}$. Third, after cooling and addition of $\mathrm{HCl}$ to reach $\mathrm{pH}=3$, the crude was extracted several time with $\mathrm{CH}_{2} \mathrm{Cl}_{2}$ (poor solubility) before to be dried under $\mathrm{MgSO}_{4}$ and filtered. Finally, after solvent removing under vacuum, the final compound 9 was isolated by column chromatography $\left(\mathrm{PE} / \mathrm{CH}_{2} \mathrm{Cl}_{2}\right.$ 9/1) and recrystallized into ethanol.

Yield: $664 \mathrm{mg}$ (52\%). ${ }^{1} \mathrm{H}$ NMR (CDCl $\left.3,300 \mathrm{MHz}\right): \delta 7.24(\mathrm{~d}, 4 \mathrm{H}), 3.71(\mathrm{~d}, 4 \mathrm{H}), 1.73(\mathrm{t}, 2 \mathrm{H})$.

\section{Compound C.}

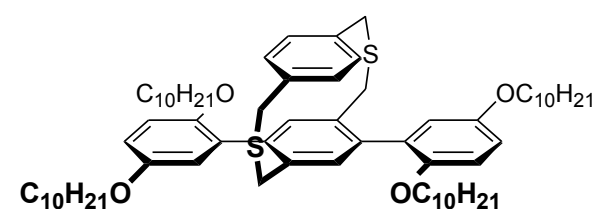

Solution (23.0 $\mathrm{mL})$ of $\mathbf{8}(67 \quad \mathrm{mg}, \quad 0.064 \mathrm{mmol})$ and 1,4-benzenedimethanethiol 9 (11 $\mathrm{mg}, 0.064 \mathrm{mmol}$ ) in $\mathrm{CH}_{2} \mathrm{Cl}_{2}$ was added drop wise to a stirred methanol solution $(20.0 \mathrm{~mL})$ of $\mathrm{KOH}(5$ $\mathrm{mg}, 0.089 \mathrm{mmol}$ ) during $6 \mathrm{~h}$. After addition of a half volume of the $\mathrm{CH}_{2} \mathrm{Cl}_{2}$ solution a second portion of $\mathrm{KOH}(4 \mathrm{mg}, 0.071 \mathrm{mmol})$ was added to the methanol solution. The solvents were evaporated, water $(5 \mathrm{~mL})$ and $\mathrm{CH}_{2} \mathrm{Cl}_{2}(5 \mathrm{~mL})$ were added, and the product was extracted into $\mathrm{CH}_{2} \mathrm{Cl}_{2}$; the combined $\mathrm{CH}_{2} \mathrm{Cl}_{2}$ extracts were dried over $\mathrm{MgSO}_{4}$, filtered, and concentrated under vacuum. The crude product was purified by column chromatography $\left(\mathrm{PE} / \mathrm{CH}_{2} \mathrm{Cl}_{2}\right.$, $9 / 1)$ to yield compound $C$ as a colorless oil.

Yield: $9 \mathrm{mg}$ (13\%). $R_{f} 0.14$ (PE/ $\left.\mathrm{CH}_{2} \mathrm{Cl}_{2}: 4 / 1\right) ;{ }^{1} \mathrm{H}$ NMR ( $\mathrm{C}_{6} \mathrm{D}_{6}, 500 \mathrm{MHz}$ ): $\delta 7.59$ (bs, 2H), 7.37 (s, 2H), 7.17 (bd, $2 \mathrm{H}, J=3.1 \mathrm{~Hz}), 7.01(\mathrm{dd}, 2 \mathrm{H}, J=3.1 \mathrm{~Hz}, J=0.6 \mathrm{~Hz}), 6.96(\mathrm{dd}, 2 \mathrm{H}, J=3.5 \mathrm{~Hz}, J=1.2 \mathrm{~Hz}), 6.80(\mathrm{~d}, 2 \mathrm{H}, J=3.5 \mathrm{~Hz})$, $4.10(\mathrm{bt}, 4 \mathrm{H}, J=2.3 \mathrm{~Hz}), 4.00(\mathrm{~d}, 2 \mathrm{H}, J=6.4 \mathrm{~Hz}), 3.96(\mathrm{~d}, 2 \mathrm{H}, J=6.4 \mathrm{~Hz}), 3.63-3.57(\mathrm{~m}, 2 \mathrm{H}), 3.45(\mathrm{~d}, 2 \mathrm{H}, J$ $=5.8 \mathrm{~Hz}), 3.46-3.38(\mathrm{~m}, 2 \mathrm{H}), 3.30(\mathrm{~d}, 2 \mathrm{H}, J=5.8 \mathrm{~Hz}), 1.91-1.82(\mathrm{~m}, 4 \mathrm{H}), 1.59-1.49(\mathrm{~m}, 4 \mathrm{H}), 1.45-1.07$ $(\mathrm{m}, 56 \mathrm{H}), 0.93-0.86(\mathrm{~m}, 12 \mathrm{H}) ;{ }^{13} \mathrm{C}$ NMR $\left(\mathrm{C}_{6} \mathrm{D}_{6}, 125 \mathrm{MHz}\right): \delta 155.3,151.6,137.2,136.4,133.5,132.5,130.1$, 129.5, 128.8, 118.8, 116.1, 115.3, 39.1, 37.5, 32.9, 30.7-30.2, 27.3, 26.9, 23.6, 14.8. HRMS (ESI): calcd. for $\mathrm{C}_{68} \mathrm{H}_{104} \mathrm{O}_{4} \mathrm{~S}_{2} \mathrm{Na}[\mathrm{M}+\mathrm{Na}]^{+}:$1071.7268; found: 1071.7249 (+1.8 ppm). calcd. for $\mathrm{C}_{68} \mathrm{H}_{104} \mathrm{O}_{4} \mathrm{~S}_{2} \mathrm{~K}[\mathrm{M}+\mathrm{K}]^{+}: 1087.7008$; found: $1087.6992(+1.5 \mathrm{ppm})$. 


\section{Dimethyl 2,5-bis(bromomethyl)terephthalate (10). ${ }^{16}$}

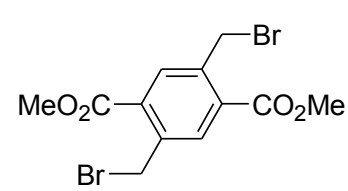

A mixture of dimethyl-2,5-dimethylterephthalate $(10.004 \mathrm{~g}, 45 \mathrm{mmol}, 1 \mathrm{eq}), \mathrm{NBS}$ (17.647g, 99mmol, 2.2eq), AIBN $(500 \mathrm{mg})$ in $\mathrm{CH}_{3} \mathrm{CN}(150 \mathrm{~mL})$ was stirred for $6 \mathrm{~h}$ at reflux. After cooling the solvent was removed under vacuum. The crude material was extracted with $\mathrm{CH}_{2} \mathrm{Cl}_{2}$, washed with brine and the combined organic layers were dried over $\mathrm{MgSO}_{4}$, filtered and concentrated under vacuum. Purification by flash chromatography (1:1 $\mathrm{CH}_{2} \mathrm{Cl}_{2}: \mathrm{PE}$ ) and recrystallization from EtOH gave 10 as a white crystalline solid.

Yield: $9.827 \mathrm{~g}(57 \%) . R_{f} 0.75(\mathrm{CH} 2 \mathrm{Cl} 2) ;{ }^{1} \mathrm{H}$ NMR $\left(\mathrm{CDCl}_{3}, 200 \mathrm{MHz}\right): \delta 3.98(\mathrm{~s}, 6 \mathrm{H}), 4.93(\mathrm{~s}, 4 \mathrm{H}), 8.05(\mathrm{~s}, 2 \mathrm{H}) ;{ }^{13} \mathrm{C}$ NMR $\left(\mathrm{CDCl}_{3}, 125 \mathrm{MHz}\right): \delta 31.8,52.4,130.0,133.8,138.9,165.9$; Anal. calcd for $\mathrm{C}_{12} \mathrm{H}_{12} \mathrm{Br}_{2} \mathrm{O}_{4}$ : $\mathrm{C}, 37.93 ; \mathrm{H}, 3.18$; $\mathrm{Br}, 42.05 ; \mathrm{O}, 16.84$; found: $\mathrm{C}, 37.73 ; \mathrm{H}, 3.24 ; \mathrm{O}, 15.79 ; \mathrm{MS} \mathrm{m} / \mathrm{z}$ : $[\mathrm{M}+\mathrm{Na}]+$ calcd: 402.89742; found: 402.89749 .

\section{Compound $11 .^{20}$}

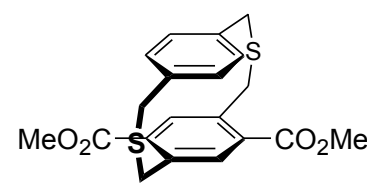

Solution (110.0 mL) of compound $10 \quad(670 \mathrm{mg}, 1.763 \mathrm{mmol})$ and 1,4-dimercaptomethylbenzene 9 (300 $\mathrm{mg}, 1.763 \mathrm{mmol}$ ) in $\mathrm{CH}_{2} \mathrm{Cl}_{2}$ was added drop wise to a stirred methanol solution $(160.0 \mathrm{~mL})$ of $\mathrm{KOH}(247 \mathrm{mg}, 4.407 \mathrm{mmol})$ during $8 \mathrm{~h}$. Then, the solvents were evaporated, water $(25 \mathrm{~mL})$ and $\mathrm{CH}_{2} \mathrm{Cl}_{2}(25 \mathrm{~mL})$ were added, and the product was extracted into $\mathrm{CH}_{2} \mathrm{Cl}_{2}$, the combined $\mathrm{CH}_{2} \mathrm{Cl}_{2}$ extracts were dried over $\mathrm{MgSO}_{4}$, filtered, and concentrated under vacuum. The crude product was purified by column chromatography $\left(\mathrm{PE} / \mathrm{CH}_{2} \mathrm{Cl}\right.$, 9/1) to yield compound 11 as a white solid after recrystallization into dried EtOAc.

Yield: $377 \mathrm{mg}(55 \%) .{ }^{1} \mathrm{H} \mathrm{NMR}\left(200 \mathrm{MHz}, \mathrm{CDCl}_{3}\right): \delta 7.46(\mathrm{~s}, 2 \mathrm{H}), 6.92(\mathrm{~m}, 4 \mathrm{H}), 3.94(6 \mathrm{H}, \mathrm{s}), 4.17(4 \mathrm{H}, \mathrm{q}, J=14$ $\mathrm{Hz}), 3.77(4 \mathrm{H}, \mathrm{q}, J=14 \mathrm{~Hz})$.

\section{Compound E.}

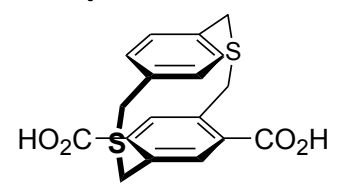

To the solution of $11(100 \mathrm{mg}, 0.257 \mathrm{mmol})$ in THF $(3.0 \mathrm{~mL})$ was added solution of $\mathrm{KOH}(288 \mathrm{mg}, 5.14 \mathrm{mmol})$ in water $(1.5 \mathrm{~mL})$. After stirring of the reaction mixture at room temperature during 7 days, solvents were evaporated. The crude product was dissolved in water and the $\mathrm{pH}$ was adjusted till $\mathrm{pH} 1$ with $\mathrm{HCl} 6 \mathrm{M}$. The reaction mixture was stirred at room temperature during $2 \mathrm{~h}$ then the precipitate was filtered and washed with water twice to give the product $\mathrm{E}$ as a white solid.

Yield: $93 \mathrm{mg}$ (99\%). mp: $248^{\circ} \mathrm{C}$; ${ }^{1} \mathrm{H}$ NMR (DMSO, $200 \mathrm{MHz}$ ): $\delta 13.06$ (bs, 2H), 7.45 (s, 2H), 6.86 (d, 2H, J $=6.9 \mathrm{~Hz}), 6.82(\mathrm{~d}, 2 \mathrm{H}, J=6.9 \mathrm{~Hz}), 4.57(\mathrm{~d}, 2 \mathrm{H}, J=14.4 \mathrm{~Hz}), 3.78(\mathrm{~s}, 4 \mathrm{H}), 3.74(\mathrm{~d}, 2 \mathrm{H}, J=14.4 \mathrm{~Hz}) .{ }^{13} \mathrm{C} \mathrm{NMR}$ (DMSO, $50 \mathrm{MHz}$ ): $\delta$ 168.0, 137.3, 135.4, 133.9, 133.2, 129.7, 129.1, 37.1, 35.0. HRMS (ESI): calcd. for $\mathrm{C}_{18} \mathrm{H}_{15} \mathrm{O}_{4} \mathrm{~S}_{2}[\mathrm{M}-\mathrm{H}]:$ : 359.0417; found: 359.0431 (-3.9 ppm).

\section{5-Bromo-1,3-phenylene)dimethanethiol (12).}<smiles>SCc1cc(Br)cc(CS)c1</smiles>

To a suspension of 1-bromo-3,5-bis(bromomethyl)benzene $(17.910,52.7 \mathrm{mmol}, 1 \mathrm{eq})$ in $\mathrm{EtOH}(200 \mathrm{~mL})$ was added thiourea $(8.393 \mathrm{~g}, 110.4 \mathrm{mmol}, 2.1 \mathrm{eq})$. The reaction mixture was refluxed overnight, then $\mathrm{EtOH}$ was removed under reduced pressure, and remaining crude intermediate was obtained. A solution of $\mathrm{KOH}(6.184 \mathrm{~g}, 110.4 \mathrm{mmol}, 2.1 \mathrm{eq})$ in $200 \mathrm{~mL}$ of water was added and the mixture was refluxed overnight. After cooling to room temperature, diluted $\mathrm{HCl}(1 \mathrm{M})$ was added to neutralize the solution to $\mathrm{pH}=1$. The aqueous solution was extracted with $\mathrm{CH}_{2} \mathrm{Cl}_{2}$, washed with brine and the combined organic layers were dried over $\mathrm{MgSO}_{4}$, filtered and concentrated under vacuum. The crude was purified by flash chromatography $\left(9: 1 \mathrm{PE}: \mathrm{CH}_{2} \mathrm{Cl}_{2}\right)$ to afford 15 as a colorless oil.

Yield: $10.650 \mathrm{~g}(81 \%) . R_{f} 0.16$ (PE); ${ }^{1} \mathrm{H} \mathrm{NMR}(200 \mathrm{MHz}, \mathrm{CDCl} 3) \delta: 1.78(\mathrm{t}, J=7.7 \mathrm{~Hz}, 2 \mathrm{H}, \mathrm{SH}), 3.68(\mathrm{~d}, J=7.7 \mathrm{~Hz}$, $\left.4 \mathrm{H}, \mathrm{CH}_{2}\right), 7.21(\mathrm{~s}, 1 \mathrm{H}), 7.36(\mathrm{~s}, 2 \mathrm{H}) ;{ }^{13} \mathrm{C} \mathrm{NMR}\left(200 \mathrm{MHz}, \mathrm{CDCl}_{3}\right) \delta: 28.46,122.78,126.66,129.97,143.75$.

\section{Compound $13 .{ }^{12}$}

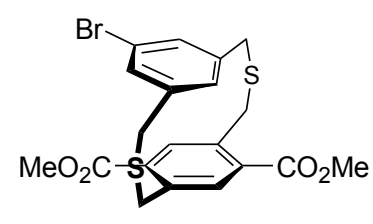

A solution of bromide compound 10 (1eq) and thiol compound 12 (1eq) in dry $\mathrm{CH}_{2} \mathrm{Cl}_{2}(50 \mathrm{~mL})$ and a solution of $\mathrm{KOH}(2.5 \mathrm{eq})$ in dry $\mathrm{MeOH}(50 \mathrm{~mL})$ were added dropwise into dry $\mathrm{MeOH}(60 \mathrm{~mL}$ per mmol of starting materials) under argon at room temperature. The addition was finished in $24 \mathrm{~h}$. The resulting mixture was neutralized with $50 \%$ sulfuric acid until $\mathrm{pH}=2$ and all the solvents were evapo- 
rated under reduced pressure. The remaining solid was extracted by $\mathrm{CH}_{2} \mathrm{Cl}_{2}$, washed with water, brine, dried other $\mathrm{MgSO}_{4}$, filtered and concentrated. The crude was purified by flash chromatography $(\mathrm{PE} / \mathrm{CH} 2 \mathrm{Cl} 2$ : $1 / 1)$ to give 13 as a white solid.

Yield: $1.371 \mathrm{~g}$ (59\%). $R_{f} 0.35$ (PE/CH $\left.\mathrm{Cl}_{2}: 1 / 1\right) ;{ }^{1} \mathrm{H}$ NMR $\left(200 \mathrm{MHz}, \mathrm{CDCl}_{3}\right) \delta: 3.48$ (q, J=15.4 Hz, 4H), 3.66 (d, $J=13.2 \mathrm{~Hz}, 2 \mathrm{H}), 3.94\left(\mathrm{~s}, 6 \mathrm{H}, \mathrm{CH}_{3}\right), 4.73(\mathrm{~d}, J=13.2 \mathrm{~Hz}, 2 \mathrm{H}), 5.71(\mathrm{~s}, 1 \mathrm{H}), 7.15(\mathrm{~s}, 2 \mathrm{H}), 7.45(\mathrm{~s}, 2 \mathrm{H}) ;{ }^{13} \mathrm{C} \mathrm{NMR}$ $\left(200 \mathrm{MHz}, \mathrm{CDCl}_{3}\right)$ ): 34.58, 34.94, 52.78, 122.64, 124.99, 130.40, 132.54, 133.39, 139.52, 151.94, 166.82; Anal. calcd for $\mathrm{C}_{20} \mathrm{H}_{19} \mathrm{O}_{4} \mathrm{~S}_{2} \mathrm{Br}$ : C, 51.39; $\mathrm{H}, 4.10 ; \mathrm{O}, 13.69 ; \mathrm{S}, 13.72 ; \mathrm{Br}, 17.10$ found: $\mathrm{C}, 51.66 ; \mathrm{H}, 4.28 ; \mathrm{O}, 13.56$; S, 13.57.

4-Hydroxyphenyl boronic acid pinacol ester (14). Commercially available

\section{Methyl 11-(4-(4,4,5,5-tetramethyl-1,3,2-dioxaborolan-2-yl)phenoxy)undecanoate (15).}<smiles>COC(=O)Oc1ccc(B2OC(C)(C)C(C)(C)O2)cc1</smiles>
A mixture of commercially available 4-hydroxyphenyl boronic acid pinacol ester $14(2.200 \mathrm{~g}, 10 \mathrm{mmol}, 1 \mathrm{eq})$, methyl 11-bromoundecanoate $(2.780 \mathrm{~g}$, $10 \mathrm{mmol}, 1 \mathrm{eq})$ and $\mathrm{K}_{2} \mathrm{CO}_{3}(2.070 \mathrm{~g}, 15 \mathrm{mmol}, 1.5 \mathrm{eq})$ in dry DMF $(20 \mathrm{~mL})$ was refluxed overnight. The resulting mixture was cooled down to room temperature and then extracted with $\mathrm{CH}_{2} \mathrm{Cl}_{2}$, washed with brine, dried over $\mathrm{MgSO}_{4}$, filtered and concentrated under vacuum. The residue was purified by flash chromatography $\left(\mathrm{CH}_{2} \mathrm{Cl}_{2}\right)$ to give 15 as a white solid.

Yield: $3.632 \mathrm{~g}(87 \%) \cdot R_{f} 0.42\left(\mathrm{CH}_{2} \mathrm{Cl}_{2}\right) ;{ }^{1} \mathrm{H}$ NMR $\left(200 \mathrm{MHz}, \mathrm{CDCl}_{3}\right) \delta: 1.30(\mathrm{~s}, 12 \mathrm{H}), 1.33(\mathrm{~s}, 12 \mathrm{H}), 1.62$ (quin, $J=7.0 \mathrm{~Hz}, 2 \mathrm{H}$ ), 1.77 (quin, $J=7.0 \mathrm{~Hz}, 2 \mathrm{H}$ ), $2.30(\mathrm{t}, J=7.0 \mathrm{~Hz}, 2 \mathrm{H}$ ), $3.66(\mathrm{~s}, 3 \mathrm{H}), 3.97(\mathrm{t}, J=7.0 \mathrm{~Hz}, 2 \mathrm{H}), 6.88(\mathrm{~d}, J$ $=8.0 \mathrm{~Hz}, 2 \mathrm{H}), 7.73(\mathrm{~d}, J=8.0 \mathrm{~Hz}, 2 \mathrm{H}) ;{ }^{13} \mathrm{C}$ NMR $\left(200 \mathrm{MHz}, \mathrm{CDCl}_{3}\right) \delta: 25.0,25.1 / 26.2 / 29.3 / 29.4 / 29.5 / 29.6,34.3$, 51.6, 68.0, 83.7, 114.0, 136.6, 161.9, 174.5); Anal. calcd for $\mathrm{C}_{24} \mathrm{H}_{39} \mathrm{BO}_{5}$ : C, 68.90; $\mathrm{H}, 9.40 ; \mathrm{O}, 19.12 ; \mathrm{B}, 2.58$ found: $C, 69.13 ; H, 9.66$.

\section{Compound 16.}

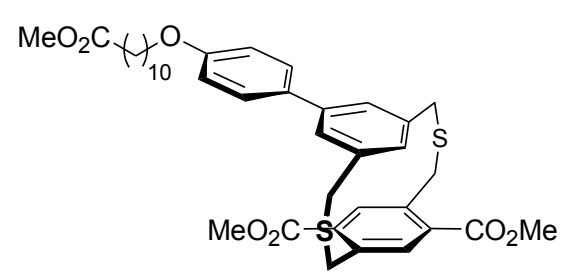

Mixture oftoluene/EtOH/ $\mathrm{H}_{2} \mathrm{O}$ was degassed for $1 \mathrm{~h}$ and then added to a stirred, degassed mixture of palladium acetate $(0.05 \mathrm{eq})$ and triphenylphosphine (0.2eq). The catalytic solution was added to a stirred, degassed mixture of bromide compound $\mathbf{1 3}$, boronic compound $\mathbf{1 5}$ $(1,5 e q)$ and sodium carbonate (20eq). The resulting mixture was refluxed for $24 \mathrm{~h}$ and cooled down to room temperature. Water was added, and the crude product was extracted with $\mathrm{CH}_{2} \mathrm{Cl}_{2}$ and washed with brine. The combined organic layers were dried over $\mathrm{MgSO}_{4}$, filtered and concentrated under vacuum. Purification by flash chromatography (PE/EtOAc: 9/1) gave 16 as a colorless oil (65mg, 48\%).

Yield: $65 \mathrm{mg}$ (48\%). $R_{f} 0.36$ (PE/EtOAc). ${ }^{1} \mathrm{H}$ NMR $\left(200 \mathrm{MHz}, \mathrm{CDCl}_{3}\right) \delta: 1.30(\mathrm{~m}, 10 \mathrm{H}), 1.47$ (quint, $\left.J=7.5 \mathrm{~Hz}, 2 \mathrm{H}\right)$, 1.61 (quint, $J=7.5 \mathrm{~Hz}, 2 \mathrm{H}), 1.79$ (quint, $J=7.5 \mathrm{~Hz}, 2 \mathrm{H}), 2.29(\mathrm{t}, J=7.5 \mathrm{~Hz}, 2 \mathrm{H}), 3.57(\mathrm{q}, J=13.2 \mathrm{~Hz}, 4 \mathrm{H}), 3.65(\mathrm{~s}$, $3 \mathrm{H}), 3.69(\mathrm{~d}, J=13.2 \mathrm{~Hz}, 2 \mathrm{H}), 3.69(\mathrm{~s}, 6 \mathrm{H}), 3.98(\mathrm{t}, J=7.5 \mathrm{~Hz}, 2 \mathrm{H}), 4.74(\mathrm{~d}, J=13.2 \mathrm{~Hz}, 2 \mathrm{H}), 5.73(\mathrm{~s}, 1 \mathrm{H}), 6.94(\mathrm{~d}$, $J=9.0 \mathrm{~Hz}, 2 \mathrm{H}), 7.21(\mathrm{~s}, 2 \mathrm{H}), 7.44(\mathrm{~s}, 2 \mathrm{H}), 7.48(\mathrm{~d}, J=10.5 \mathrm{~Hz}, 2 \mathrm{H}) .{ }^{13} \mathrm{C} \mathrm{NMR}\left(200 \mathrm{MHz}, \mathrm{CDCl}_{3}\right) \delta$ : 25.0/29.2/29.3/29.4/29.4/29.4/29.5, 34.2, 34.6, 35.5, 51.5, 52.3, 68.2, 114.7, 124.7, 125.6, 128.0, 132.2, 133.2, 133.4, 139.6, 140.0, 140.9, 158.8, 166.7, 174.3. Anal. calcd for $\mathrm{C}_{38} \mathrm{H}_{46} \mathrm{O}_{7} \mathrm{~S}_{2}$ : C, 67.23; $\mathrm{H}, 6.83 ; \mathrm{O}, 16.50$; S, 9.45; found: $\mathrm{C}, 67.45 ; \mathrm{H}, 6.77 ; \mathrm{O}, 16.29 ; \mathrm{S}, 9.49 . \mathrm{MS} \mathrm{m} / \mathrm{z}$ : $[\mathrm{M}+\mathrm{Na}]^{+}$calcd: 701.25772 ; found: 701.25789

\section{Compound G.}

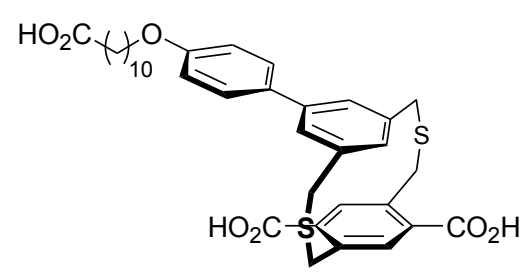

To a solution of compound $16(88 \mathrm{mg}, 0.130 \mathrm{mmol})$ in THF $(3.0 \mathrm{~mL})$ was added solution of $\mathrm{KOH}(100 \mathrm{mg}, 1.786 \mathrm{mmol}, 13 \mathrm{eq})$ in water $(1.5 \mathrm{~mL})$. The mixture was stirred at room temperature under argon atmosphere for 9 days, and then all the solvents were evaporated under reduced pressure. The crude product was dissolved in water and the $\mathrm{pH}$ was adjusted till $\mathrm{pH}=1$ with $\mathrm{HCl} 6 \mathrm{M}$. The reaction mixture was stirred at room temperature during $2 \mathrm{~h}$. The resulting mixture was filtered, the precipitate was washed with water and then dried over high vacuum to afford $\mathbf{G}$ as a white solid (58mg, $70 \%$ ).

Yield: $58 \mathrm{mg}(70 \%) .{ }^{1} \mathrm{H}$ NMR $\left(200 \mathrm{MHz}, \mathrm{DMSO}-\mathrm{D}_{6}\right) \delta: 1.27(\mathrm{~m}, 12 \mathrm{H}), 1.46$ (quint, $J=7.5 \mathrm{~Hz}, 2 \mathrm{H}$ ), 1.73 (quint, $J=7.5 \mathrm{~Hz}, 2 \mathrm{H}), 2.19(\mathrm{t}, \mathrm{t}, J=7.5 \mathrm{~Hz}, 2 \mathrm{H}), 3.57(\mathrm{q}, J=13.2 \mathrm{~Hz}, 4 \mathrm{H}), 3.80(\mathrm{~d}, J=13.2 \mathrm{~Hz}, 2 \mathrm{H}), 3.99(\mathrm{t}, J=7.5 \mathrm{~Hz}$, 
2H), $4.61(\mathrm{~d}, J=13.2 \mathrm{~Hz}, 2 \mathrm{H}), 5.60(\mathrm{~s}, 1 \mathrm{H}), 6.97(\mathrm{~d}, J=8.7 \mathrm{~Hz}, 2 \mathrm{H}), 7.06(\mathrm{~s}, 2 \mathrm{H}), 7.41(\mathrm{~d}, J=8.7 \mathrm{~Hz}, 2 \mathrm{H}), 7.52(\mathrm{~s}$, 2H). ${ }^{13} \mathrm{C}$ NMR (200MHz, DMSO-D 6 ) $\delta: ~ 24.3 / 25.5 / 28.5 / 28.7 / 28.8 / 28.8 / 28.9,33.6,33.6,34.1,67.5,114.6$, $124.4,125.1,127.8,133.0,133.1,133.1,138.6,140.0,140.2,158.0,167.8,174.5$. Anal. calcd for $\mathrm{C}_{35} \mathrm{H}_{40} \mathrm{O}_{7} \mathrm{~S}_{2}$ : C, 66.01; H, 6.33; O, 17.59; S, 10.07; found: C, 65.90; H, 6.38; S, 9.85. MS m/z: [M+Na] ${ }^{+}$calcd: 659.21077; found: 659.21028 .

Methyl4-(4,4,5,5-tetramethyl-1,3,2-dioxaborolan-2yl)benzoate (17). Commercially available.

dimethyl 2',5'-dimethyl-[1,1':4',1"-terphenyl]-4,4"-dicarboxylate (18).

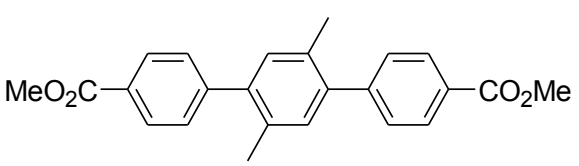

Degassed THF $(3.0 \mathrm{~mL})$ and water $(1.5 \mathrm{~mL})$ were added to a stirred, degassed mixture of methyl-4-(4,4,5,5-tetramethyl-1,3,2dioxaborolan-yl)benzoate 17 (400 mg, 1,53 mmol), 1,4-dibromo-2,5dimethylbenzene ( $183 \mathrm{mg}, 0.694 \mathrm{mmol})$, potassium carbonate $(422$ $\mathrm{mg}, 3.05 \mathrm{mmol}$ ) and tetrakis(triphenylphosphine)palladium(0) $(40 \mathrm{mg}, 0.0345 \mathrm{mmol})$. The mixture was heated under reflux for one night and cooled. Water was added, and the crude product was extracted into $\mathrm{CH}_{2} \mathrm{Cl}_{2}$. The combined $\mathrm{CH}_{2} \mathrm{Cl}_{2}$ extracts were washed with brine and dried $\left(\mathrm{MgSO}_{4}\right)$. The desiccant was filtered off, the solvent was removed in vacuo, and the crude product was purified by column chromatography (PE/ $\mathrm{CH}_{2} \mathrm{Cl}_{2}$ ) to give the product 18 as a white solid.

Yield: $216 \mathrm{mg}$ (83\%). $R_{f} 0.49$ (PE/ $\left.\mathrm{CH}_{2} \mathrm{Cl}_{2}: 1 / 1\right)$. $\mathrm{mp}: 86^{\circ} \mathrm{C} .{ }^{1} \mathrm{H}$ NMR $\left(\mathrm{CDCl}_{3}, 500 \mathrm{MHz}\right)$ :

$\delta 8.11(\mathrm{~d}, 4 \mathrm{H}, \mathrm{J}=8.3 \mathrm{~Hz}), 7.45(\mathrm{~d}, 4 \mathrm{H}, \mathrm{J}=8.3 \mathrm{~Hz}), 7.16(\mathrm{~s}, 2 \mathrm{H}), 3.96(\mathrm{~s}, 6 \mathrm{H}), 2.28(\mathrm{~s}, 6 \mathrm{H}) .{ }^{13} \mathrm{C}-\mathrm{NMR}\left(\mathrm{CDCl}_{3}, 125\right.$ $\mathrm{MHz}): \delta=167.1,146.4,140.5,132.8,131.8,129.6,129.4,128.8,52.2,19.9$.

\section{Compound D.}

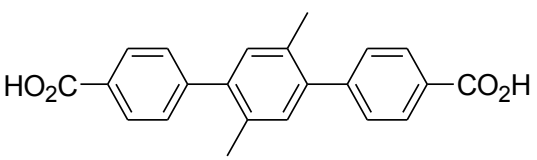

To the solution of compound 18 (154 mg, $0.411 \mathrm{mmol})$ in THF $(4.0 \mathrm{~mL})$ was added solution of $\mathrm{KOH}(231 \mathrm{mg}, 4.113 \mathrm{mmol})$ in water $(2.0 \mathrm{~mL})$. After stirring of the reaction mixture at room temperature during 2 days solvents were evaporated. The crude product was dissolved in water and the $\mathrm{pH}$ was adjusted till $\mathrm{pH} 1$ with $\mathrm{HCl} 6 \mathrm{M}$. The reaction mixture was stirred at room temperature during $2 \mathrm{~h}$ then the precipitate was filtered and washed with water twice to give the product $\mathbf{D}$ as a white solid.

Yield: $142 \mathrm{mg}$ (99\%). mp: $163^{\circ} \mathrm{C} .{ }^{1} \mathrm{H}$ NMR (DMSO, $200 \mathrm{MHz}$ ): $\delta 12.99$ (bs, 2H), 8.02 (d, $4 \mathrm{H}, J=8.4 \mathrm{~Hz}$ ), 7.52 (d, $4 \mathrm{H}, J=8.4 \mathrm{~Hz}$ ), $7.20(\mathrm{~s}, 2 \mathrm{H}), 2.25(\mathrm{~s}, 6 \mathrm{H}) .{ }^{13} \mathrm{C}$ NMR (DMSO, $\left.50 \mathrm{MHz}\right): \delta 167.2,145.3,139.8,132.3,131.6$, 129.3, 129.3, 129.2, 19.5. HRMS (ESI): calcd. for $\mathrm{C}_{22} \mathrm{H}_{17} \mathrm{O}_{4}[\mathrm{M}-\mathrm{H}]: 345.1132$; found: 345.1135 (-0.9 ppm).

\section{dimethyl 2',5'-bis(methoxymethyl)-[1,1':4',1'-terphenyl]-4,4"'-dicarboxylate (19).}

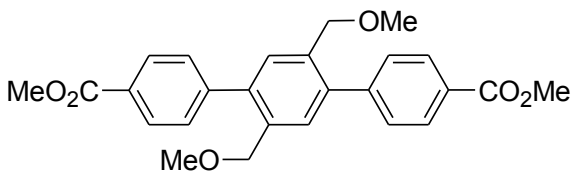

Degassed THF $(14.0 \mathrm{~mL})$ and water $(7.0 \mathrm{~mL})$ were added to a stirred, degassed mixture of methyl 4-(4,4,5,5-tetramethyl-1,3,2dioxaborolan-2-yl)benzoate 17 ( $2.0 \mathrm{~g}, 7.63 \mathrm{mmol}), 1,4$-dibromo-2,5bis(methoxymethyl)benzene $6(1.12 \mathrm{~g}, 3.47 \mathrm{mmol})$, potassium carbonate $(2.11 \mathrm{~g}, 15.27 \mathrm{mmol})$ and tetrakis(triphenylphosphine) palladium(0) (200 $\mathrm{mg}, 0.173 \mathrm{mmol})$. The mixture was heated under reflux for 2.5 days and cooled. Water was added, and the crude product was extracted into $\mathrm{CH}_{2} \mathrm{Cl}_{2}$. The combined $\mathrm{CH}_{2} \mathrm{Cl}_{2}$ extracts were washed with brine and dried $\left(\mathrm{MgSO}_{4}\right)$. The desiccant was filtered off, the solvent was removed in vacuo, and the crude product was purified by column chromatography (PE/ $\left.\mathrm{CH}_{2} \mathrm{Cl}_{2}: 1 / 1\right)$ to give the product 19 as a white solid.

Yield: $1.45 \mathrm{~g}$ (96\%). $R_{f} 0.59$ (cyclohexane/ $\left.\mathrm{CH}_{2} \mathrm{Cl}_{2}: 1 / 1\right)$. $\mathrm{mp}: 162^{\circ} \mathrm{C} ;{ }^{1} \mathrm{H} \mathrm{NMR}\left(\mathrm{CDCl}_{3}, 200 \mathrm{MHz}\right): \delta 8.11$ (d, $4 \mathrm{H}$, $J=8.3 \mathrm{~Hz}), 7.51(\mathrm{~d}, 4 \mathrm{H}, J=8.3 \mathrm{~Hz}), 7.48(\mathrm{~s}, 2 \mathrm{H}), 4.33(\mathrm{~s}, 4 \mathrm{H}), 3.95(\mathrm{~s}, 6 \mathrm{H}), 3.34(\mathrm{~s}, 6 \mathrm{H}) ;{ }^{13} \mathrm{C} \mathrm{NMR}\left(\mathrm{CDCl}_{3}, 50\right.$ $\mathrm{MHz}$ ): $\delta$ 167.1, 145.1, 140.6, 135.1, 131.0, 129.6, 129.4, 129.2, 72.2, 58.5, 52.3; HRMS (ESI): calcd. for $\mathrm{C}_{26} \mathrm{H}_{26} \mathrm{O}_{6} \mathrm{Na}[\mathrm{M}+\mathrm{Na}]^{+}:$457.1622; found: 457.1639 (-3.9 ppm). calcd. for $\mathrm{C}_{26} \mathrm{H}_{26} \mathrm{O}_{6} \mathrm{~K}[\mathrm{M}+\mathrm{K}]^{+}:$: 473.1361; found: $473.1368(-1.6 \mathrm{ppm})$.

dimethyl 2',5'-bis(bromomethyl)-[1,1':4',1"-terphenyl]-4,4"-dicarboxylate (20).

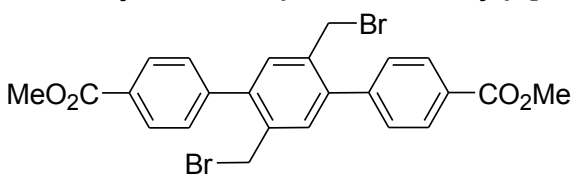

To the dry $\mathrm{CH}_{2} \mathrm{Cl}_{2}$ solution $(10.0 \mathrm{~mL})$ of compound $19(500 \mathrm{mg}$, $0.869 \mathrm{mmol}$ ) was added boron tribromide (1.0 M in $\left.\mathrm{CH}_{2} \mathrm{Cl}_{2}, 1.96 \mathrm{~mL}\right)$ under an argon atmosphere. The reaction mixture was stirred at 
room temperature under argon for $2 \mathrm{~h}$, then quenched with water at $0^{\circ} \mathrm{C}$, and extracted with $\mathrm{CH}_{2} \mathrm{Cl}_{2}$ $(3 \times 20 \mathrm{~mL})$. The organic layer was washed with brine, dried over $\mathrm{MgSO}_{4}$, filtered, and evaporated. The crude product was subsequently washed two times with methanol to give the product $\mathbf{2 0}$ as a white solid.

Yield: $462 \mathrm{mg}$ (99\%). $R_{f} 0.58$ (cyclohexane/DCM: 1/3); ${ }^{1} \mathrm{H}$ NMR $\left(\mathrm{CDCl}_{3}, 200 \mathrm{MHz}\right): \delta 8.16(\mathrm{~m}, 4 \mathrm{H}), 7.58(\mathrm{~m}$, $4 \mathrm{H}), 7.44(\mathrm{~s}, 2 \mathrm{H}), 4.42(\mathrm{~s}, 4 \mathrm{H}), 3.97(\mathrm{~s}, 6 \mathrm{H}) ;{ }^{13} \mathrm{C} \mathrm{NMR}\left(\mathrm{CDCl}_{3}, 50 \mathrm{MHz}\right): \delta 166.9,143.9,141.3,135.8,132.9$, $129.9,129.8,129.2,52.4,30.9$.

\section{Compound 21.}

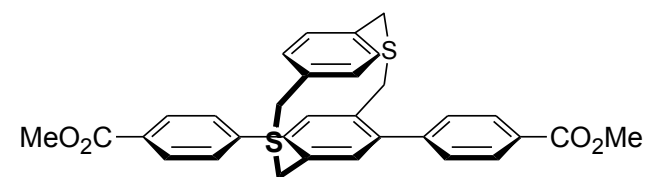

Solution $(50.0 \mathrm{~mL})$ of compound $20(300 \mathrm{mg}, 0.564 \mathrm{mmol})$ and 1,4-phenylenedimethanethiol 9 (96 $\mathrm{mg}, 0.564 \mathrm{mmol}$ ) in $\mathrm{CH}_{2} \mathrm{Cl}_{2}$ was added drop wise to a stirred methanol solution $(75.0 \mathrm{~mL})$ of $\mathrm{KOH}(40 \mathrm{mg}, 0.713 \mathrm{mmol})$ during $6 \mathrm{~h}$. After addition of a half volume of the $\mathrm{CH}_{2} \mathrm{Cl}_{2}$ solution a second portion of $\mathrm{KOH}$ ( $39 \mathrm{mg}, 0.695 \mathrm{mmol}$ ) was added to the reaction mixture (methanol solution). The solvents were evaporated, water $(30 \mathrm{~mL})$ and $\mathrm{CH}_{2} \mathrm{Cl}_{2}(30 \mathrm{~mL})$ were added, and the product was extracted into $\mathrm{CH}_{2} \mathrm{Cl}_{2}$; the combined $\mathrm{CH}_{2} \mathrm{Cl}_{2}$ extracts were dried over $\mathrm{MgSO}_{4}$, filtered, and concentrated under vacuum. The crude product was purified by column chromatography $\left(\mathrm{PE} / \mathrm{CH}_{2} \mathrm{Cl}_{2}: 1 / 1\right)$ to yield $\mathbf{2 1}$ as a colorless solid.

Yield: $187 \mathrm{mg}$ (61\%). $R_{f} 0.21$ (cyclohexane/ $\mathrm{CH}_{2} \mathrm{Cl}_{2}: 1 / 3$ ). $\mathrm{mp}: 118^{\circ} \mathrm{C} ;{ }^{1} \mathrm{H}$ NMR $\left(\mathrm{CDCl}_{3}, 200 \mathrm{MHz}\right.$ ): $\delta 8.16$ (d, $4 \mathrm{H}$, $J=8.4 \mathrm{~Hz}), 7.48(\mathrm{~d}, 4 \mathrm{H}, J=8.4 \mathrm{~Hz}), 7.28(\mathrm{~s}, 2 \mathrm{H}), 7.13(\mathrm{dd}, 2 \mathrm{H}, J=7.8 \mathrm{~Hz}, J=1.7 \mathrm{~Hz}), 6.80(\mathrm{dd}, 2 \mathrm{H}, J=7.8 \mathrm{~Hz}$, $J=1.7 \mathrm{~Hz}$ ), $3.96(\mathrm{~s}, 6 \mathrm{H}), 3.94(\mathrm{~d}, 2 \mathrm{H}, J=15.9 \mathrm{~Hz}), 3.79(\mathrm{~d}, 2 \mathrm{H}, J=15.9 \mathrm{~Hz}) 3.71(\mathrm{~d}, 2 \mathrm{H}, J=14.6 \mathrm{~Hz}), 3.49(\mathrm{~d}, 2 \mathrm{H}$, $J=14.6 \mathrm{~Hz}) ;{ }^{13} \mathrm{C} \mathrm{NMR}\left(\mathrm{CDCl}_{3}, 50 \mathrm{MHz}\right): \delta 166.9,145.0,138.9,135.9,133.5,132.5,129.8,129.3,129.2,128.9$, 128.3, 52.2, 38.4, 35.5); HRMS (ESI): calcd. for $\mathrm{C}_{64} \mathrm{H}_{56} \mathrm{O}_{8} \mathrm{~S}_{4} \mathrm{Na}[2 \times \mathrm{M}+\mathrm{Na}]^{+}: 1103.2750$; found: 1103.2726 (+2.2 ppm).

\section{Compound F.}

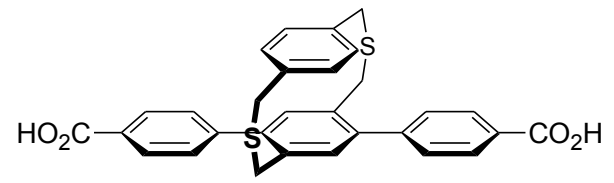

Solution of diester 21 (115 mg, $0.213 \mathrm{mmol})$ and of $\mathrm{KOH}(220 \mathrm{mg}$, $3.921 \mathrm{mmol})$ in mixture of THF $(10 \mathrm{~mL})$ and water $(6 \mathrm{~mL})$ was stirred at room temperature under argon during 7 days, then solvents were evaporated. The crude product was dissolved in water and the $\mathrm{pH}$ was adjusted till $\mathrm{pH} 1$ with $\mathrm{HCl} 6 \mathrm{M}$. The reaction mixture was stirred at room temperature during $2 \mathrm{~h}$ then the precipitate was filtered and washed with water twice to give the product $\mathbf{F}$ as a white solid.

Yield: $89 \mathrm{mg}$ (82\%). mp: $298^{\circ} \mathrm{C} ;{ }^{1} \mathrm{H}$ NMR (DMSO, $200 \mathrm{MHz}$ ): $\delta 13.05$ (s, 2H), 8.13 (d, 4H, J = 8.1 Hz), 7.65 (d, $4 \mathrm{H}, J=8.1 \mathrm{~Hz}), 7.27(\mathrm{~s}, 2 \mathrm{H}), 7.17(\mathrm{bd}, 2 \mathrm{H}, J=7.9 \mathrm{~Hz}), 6.85(\mathrm{bd}, 2 \mathrm{H}, J=7.9 \mathrm{~Hz}), 4.06(\mathrm{~d}, 2 \mathrm{H}, J=15.1 \mathrm{~Hz}), 3.95$ $\left(\mathrm{d}, 2 \mathrm{H}, J=15.8 \mathrm{~Hz}\right.$ ), $3.81(\mathrm{~d}, 2 \mathrm{H}, J=14.4 \mathrm{~Hz}), 3.60\left(\mathrm{~d}, 2 \mathrm{H}, J=14.5 \mathrm{~Hz}\right.$ ); ${ }^{13} \mathrm{C}$ NMR (DMSO, $\left.50 \mathrm{MHz}\right): \delta 168.3$, $145.8,139.2,136.6,134.3,133.4,130.5,130.2,130.1,38.0,35.8$. HRMS (ESI): calcd. for $\mathrm{C}_{26} \mathrm{H}_{26} \mathrm{O}_{6}$ [M-H]: 457.1622; found: 457.1639 (-3.9 ppm).

\section{Compound 22.}

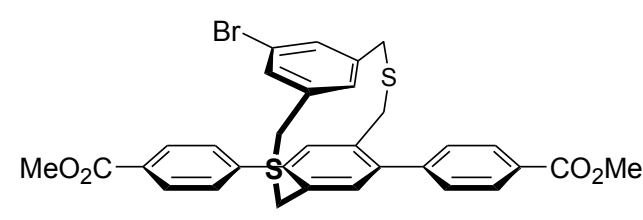

A solution of bromide compound 20 (1eq) and thiol compound 12 (1eq) in dry $\mathrm{CH}_{2} \mathrm{Cl}_{2}(50 \mathrm{~mL})$ and a solution of $\mathrm{KOH}$ (2.5eq) in dry $\mathrm{MeOH}(50 \mathrm{~mL})$ were added dropwise into dry $\mathrm{MeOH}(60 \mathrm{~mL}$ per $\mathrm{mmol}$ of starting materials) under argon at room temperature. The addition was finished in $24 \mathrm{~h}$. The resulting mixture was neutralized with $50 \%$ sulfuric acid until $\mathrm{pH}=2$ and all the solvents were evaporated under reduced pressure. The remaining solid was extracted by $\mathrm{CH}_{2} \mathrm{Cl}_{2}$, washed with water, brine, dried other $\mathrm{MgSO}_{4}$, filtered and concentrated. Purification by flash chromatography $\left(\mathrm{PE} / \mathrm{CH}_{2} \mathrm{Cl}_{2}: 1 / 1\right)$ gave 22 as a white solid.

Yield: $360 \mathrm{mg}(58 \%) . \mathrm{mp}: 298^{\circ} \mathrm{C} ; R_{f} 0.53\left(\mathrm{CH}_{2} \mathrm{Cl}_{2}\right) .{ }^{1} \mathrm{H} \mathrm{NMR}\left(200 \mathrm{MHz}, \mathrm{CDCl}_{3}\right) \delta: 3.40(\mathrm{q}, \mathrm{J}=15.2 \mathrm{~Hz}, 4 \mathrm{H}), 3.79$ $(\mathrm{d}, \mathrm{J}=13.8 \mathrm{~Hz}, 2 \mathrm{H}), 3.97(\mathrm{~s}, 3 \mathrm{H}), 4.15(\mathrm{~d}, \mathrm{~J}=13.7 \mathrm{~Hz}, 2 \mathrm{H}), 5.90(\mathrm{~s}, 1 \mathrm{H}), 7.10(\mathrm{~s}, 2 \mathrm{H}), 7.22(\mathrm{~d}, J=7.7 \mathrm{~Hz}, 4 \mathrm{H})$, $7.26(\mathrm{~s}, 2 \mathrm{H}), 8.13$ (d, $J=7.7 \mathrm{~Hz}, 4 \mathrm{H}) .{ }^{13} \mathrm{C} \mathrm{NMR}\left(200 \mathrm{MHz}, \mathrm{CDCl}_{3}\right)$ 8: 35.2, 35.2, 52.4, 122.7, 125.0, 129.4, 129.5, 130.0, 130.6, 132.9, 135.6, 140.8, 142.5, 144.7, 167.0. Anal. calcd for $\mathrm{C}_{32} \mathrm{H}_{27} \mathrm{O}_{4} \mathrm{~S} 2 \mathrm{Br}: \mathrm{C}, 62.03 ; \mathrm{H}, 4.39 ; \mathrm{O}$, 10.33 ; S, 10.35; $\mathrm{Br}, 12.90$; found: C, 62.02; H, 4.50; O, 10.10; S, 10.06 . 


\section{Compound 23.}

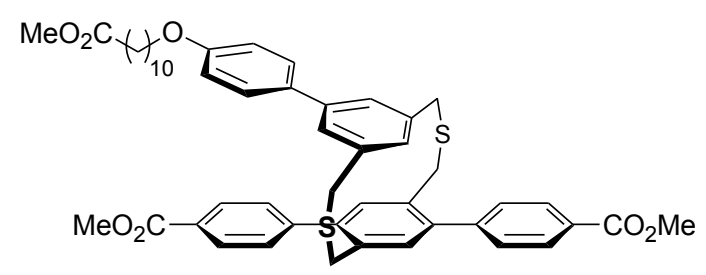

Mixture of toluene/EtOH/ $\mathrm{H}_{2} \mathrm{O}$ was degassed for $1 \mathrm{~h}$ and added to a stirred, degassed mixture of palladium acetate (0.05eq) and triphenylphosphine (0.2eq). The catalytic solution was added to a stirred, degassed mixture of bromide compound 22, boronic compound 15 (1,5eq) and sodium carbonate (20eq). The resulting mixture was refluxed for $24 \mathrm{~h}$ and cooled down to room temperature. Water was added, and the crude product was extracted with $\mathrm{CH}_{2} \mathrm{Cl}_{2}$ and washed with brine. The combined organic layers were dried over $\mathrm{MgSO}_{4}$, filtered and concentrated under vacuum. Purification by flash chromatography $\left(\mathrm{PE} / \mathrm{CH}_{2} \mathrm{Cl}_{2}: 1 / 2\right)$ gave 23 as a white solid.

Yield: $98 \mathrm{mg}(59 \%) . R_{f} 0.76\left(\mathrm{CH}_{2} \mathrm{Cl}_{2}\right) .{ }^{1} \mathrm{H}$ NMR $\left(200 \mathrm{MHz}, \mathrm{CDCl}_{3}\right) \delta: 1.32(\mathrm{~m}, 12 \mathrm{H}), 1.63$ (quint, $\left.J=7.5 \mathrm{~Hz}, 2 \mathrm{H}\right)$, 1.84 (quint, $J=7.5 \mathrm{~Hz}, 2 \mathrm{H}), 2.31(\mathrm{t}, J=7.5 \mathrm{~Hz}, 2 \mathrm{H}), 3.50(\mathrm{q}, J=13.2 \mathrm{~Hz}, 4 \mathrm{H}), 3.66(\mathrm{~s}, 3 \mathrm{H}), 3.80(\mathrm{~d}, J=13.2 \mathrm{~Hz}$, $2 \mathrm{H}), 3.95(\mathrm{~s}, 6 \mathrm{H}), 4.03(\mathrm{t}, J=7.5 \mathrm{~Hz}, 2 \mathrm{H}), 4.16(\mathrm{~d}, J=13.2 \mathrm{~Hz}, 2 \mathrm{H}), 5.88(\mathrm{~s}, 1 \mathrm{H}), 7.02(\mathrm{~d}, J=8.8 \mathrm{~Hz}, 2 \mathrm{H}), 7.13(\mathrm{~s}$, $2 \mathrm{H}), 7.21(\mathrm{~d}, J=7.7 \mathrm{~Hz}, 2 \mathrm{H}), 7.24(\mathrm{~s}, 2 \mathrm{H}), 7.52(\mathrm{~d}, J=8.7 \mathrm{~Hz}, 2 \mathrm{H}), 8.02(\mathrm{~d}, J=7.7 \mathrm{~Hz}, 2 \mathrm{H}) .{ }^{13} \mathrm{C}$ NMR $(200 \mathrm{MHz}$, $\mathrm{CDCl}_{3}$ ) $\delta:$ 25.0/26.2/29.2/29.3/29.4/29.5/29.5/29.6, 34.2, 35.3, 35.7, 51.5, 52.3, 68.2, 115.0, 124.7, 125.8, $128.1,129.1,129.4,129.8,132.9,133.0,135.6,140.7,140.7,141.2,144.9,159.0,166.9,174.4$. Anal. calcd for $\mathrm{C}_{50} \mathrm{H}_{54} \mathrm{O}_{7} \mathrm{~S}_{2}: \mathrm{C}, 72.26 ; \mathrm{H}, 6.55 ; 0,13.48 ; \mathrm{S}, 7.72 ;$ found: $\mathrm{C}, 72.37 ; \mathrm{H}, 6.59 ; 0,13.39 ; \mathrm{S}, 7.65$. MS m/z: $[\mathrm{M}+\mathrm{Na}]^{+}$calcd: 853.32032 ; found: 853.32021

\section{Compound H.}

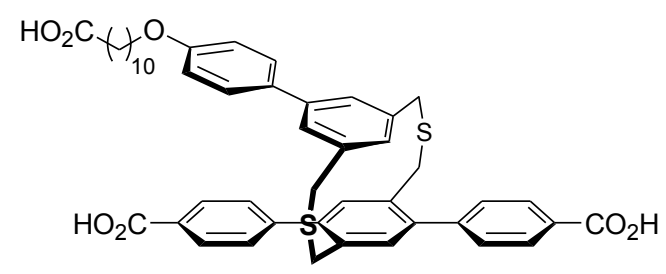

To a solution of compound $23(60 \mathrm{mg}, 0.072 \mathrm{mmol})$ in THF $(2.0 \mathrm{~mL})$ was added solution of $\mathrm{KOH}(100 \mathrm{mg}, 1.786 \mathrm{mmol})$ in water $(1.0 \mathrm{~mL})$. The mixture was stirred at room temperature under argon atmosphere for 7 days, and then all the solvents were evaporated under reduced pressure. The crude product was dissolved in water and the $\mathrm{pH}$ was adjusted till $\mathrm{pH}=1$ with $\mathrm{HCl}$ $6 \mathrm{M}$. After stirring at room temperature during $2 \mathrm{~h}$, the resulting mixture was filtered, the precipitate was washed with water and then dried over high vacuum to afford $\mathbf{H}$ as a white solid (54mg, 95\%).

Yield: $54 \mathrm{mg}(95 \%) .{ }^{1} \mathrm{H}$ NMR $\left(200 \mathrm{MHz}, \mathrm{DMSO}-\mathrm{D}_{6}\right) \delta: 1.28(\mathrm{~m}, 12 \mathrm{H}), 1.49$ (quint, $\left.J=7.5 \mathrm{~Hz}, 2 \mathrm{H}\right), 1.76$ (quint, $J=7.5 \mathrm{~Hz}, 2 \mathrm{H}), 2.19(\mathrm{t}, J=7.5 \mathrm{~Hz}, 2 \mathrm{H}), 3.60(\mathrm{q}, J=13.2 \mathrm{~Hz}, 4 \mathrm{H}), 3.80(\mathrm{~d}, J=13.2 \mathrm{~Hz}, 2 \mathrm{H}), 3.99(\mathrm{t}, J=6.4 \mathrm{~Hz}, 2 \mathrm{H})$, $4.16(\mathrm{~d}, J=13.2 \mathrm{~Hz}, 2 \mathrm{H}), 5.86(\mathrm{~s}, 1 \mathrm{H}), 7.06(\mathrm{~d}, J=8.7 \mathrm{~Hz}, 2 \mathrm{H}), 7.11(\mathrm{~s}, 2 \mathrm{H}), 7.28(\mathrm{~d}, J=7.7 \mathrm{~Hz}, 2 \mathrm{H}), 7.33(\mathrm{~s}, 2 \mathrm{H})$, $7.54(\mathrm{~d}, J=8.7 \mathrm{~Hz}, 2 \mathrm{H}), 7.89(\mathrm{~d}, J=7.7 \mathrm{~Hz}, 2 \mathrm{H}), 12.58(\mathrm{~m}, 3 \mathrm{H}) .{ }^{13} \mathrm{C} N M R\left(200 \mathrm{MHz}, \mathrm{DMSO}-\mathrm{D}_{6}\right) \delta$ : 25.0/26.2/29.2/29.3/29.4/29.5/29.5/29.6, 30.7, 35.3, 35.7, 67.0, 114.9, 124.7, 125.8, 128.1, 129.1, 129.4, $129.8,132.9,133.0,135.6,140.7,140.7,141.2,144.5,159.0,169.3,178.4$. Anal. calcd for $\mathrm{C}_{47} \mathrm{H}_{48} \mathrm{O}_{7} \mathrm{~S}_{2}: \mathrm{C}$, 71.55; H, 6.13; O, 14.19; S, 8.13; found: C, 71.67; H, 6.23; S, 8.19. MS m/z: [M-H]- calcd: 787.27687; found: 787.27431.

\section{$\underline{\text { STM }}$}

STM images were acquired at room temperature with a homemade digital system. The fast-scan axis was kept perpendicular to the sample slope. All images were obtained in the height mode, i.e. with real-time current regulation. Images acquired simultaneously in both fast scan directions were systematically recorded and compared. All images were corrected for the drift of the instrument, by combining two successive images with downward and upward slow-scan directions, using especially developed image cross-correlation software called Imago. The solvent for the self-assembly was 1-phenyloctane (98\%, Aldrich), which is well suited for in situ scanning tunneling microscopy (STM) because of its low conductivity, slow volatility and high dielectric rigidity and also avoids the coadsorption often observed with linear alkanes. The substrate was either a freshly cleaved HOPG (SPI, grade 2) or Au(111) and the tips were mechanically formed from a $250 \mathrm{~mm} \mathrm{Pt/Ir} \mathrm{wire} \mathrm{(Pt80Ir20,} \mathrm{Goodfellow).} \mathrm{The} \mathrm{freshly-cleaved} \mathrm{sample} \mathrm{and} \mathrm{tip}$ quality was systematically checked by STM observation of HOPG atomic network prior to molecular deposition. The monolayers were formed by immersing the STM junction in a droplet (ca. $5 \mu \mathrm{L}$ ) of a solution immediately after observation of HOPG atomic network. Imaging was then carried out in situ at the liquidsolid interface. 


\section{References}

(1) a) De Feyter, S.; De Schryver F.C. Two-dimensional supramolecular self-assembly probed by scanning tunneling microscopy. Chem. Soc. Rev. 2003, 32, 139-150. b) Barth, J.V. Molecular architectonic on metal surfaces. Annu. Rev. Phys. Chem. 2007, 58, 375-40. c)Theobald, J. A.; Oxtoby, N. S.; Phillips, M. A.; Champness, N. R; Beton, P. H. Controlling molecular deposition and layer structure with supramolecular surface assemblies. Nature 2003, 424, 1029-1031. d) Urdampilleta, M.; Klyatskaya, S.; Cleuziou, J-P.; Ruben, M.; Wernsdorfer, W. Supramolecular spin valves. Nature Materials 2011, 10502. e) Ozbay, E. Plasmonics: Merging Photonics and Electronics at Nanoscale Dimensions. Science 2006, 311 (5758), 189-193. f) Pillai, S.; Catchpole, K. R.; Trupke, T.; Green, M. A. Surface Plasmon Enhanced Silicon Solar Cells. J. Appl. Phys. 2007, 101 (9), 93105. g) Minh Hiep, H.; Endo, T.; Kerman, K.; Chikae, M.; Kim, D.-K.; Yamamura, S.; Takamura, Y.; Tamiya, E. A Localized Surface Plasmon Resonance Based Immunosensor for the Detection of Casein in Milk. Sci. Technol. Adv. Mater. 2007, 8 (4), 331-338. h) Mullen, K.; Rabe, J.P. Acc. Chem. Res. 2008, 41, 511. i) Mali, K.S.; Adisoejoso, J.; Ghijsens, E.; De Cat, I.; De Feyter, S. Exploring the Complexity of Supramolecular Interactions for Patterning at the Liquid/Solid Interface. Acc. Chem. Res. 2012, 45 (8), 1309-1320.

(2) a) Ivasenko, O.; Perepichka, D. Mastering fundamentals of supramolecular design with carboxylic acids. Common lessons from X-ray crystallography and scanning tunneling microscopy. Chem. Soc. Rev. 2011, 40, 191-206. b) Madueno, R.; Räisänen, M.T.; Silien, C.; Buck, M. Functionalizing hydrogenbonded surface networks with self-assembled monolayers, Nature Letter, 2008, 454, 618-621. c) Li, Z.; Han, B.; Wan, L. J.; Wandlowski, T. Supramolecular nanostructures of 1,3,5-benzene-tricarboxylic acid at electrified $\mathrm{Au}(111) / 0.05 \mathrm{M} \mathrm{H}_{2} \mathrm{SO}_{4}$ interfaces: An in situ scanning tunneling microscopy study. Langmuir 2005, 21, 6915-6928. d) Pinheiro, L. S.; Temperini, M. L. A. Pyridine and pyridine carboxylic acids as guests in a bidimensional hydrogen bond structure analyzed by scanning tunneling microscopy. Surf. Sci. 2007, 601, 1836-1843. e) Kampschulte, L.; Griessl, S.; Heckl, W. M.; Lackinger, M. Mediated coadsorption at the liquid-solid interface: Stabilization through hydrogen bonds. J. Phys. Chem. 2005, B 109, 14074-14078.

(3) a) Furukawa, S.; Tahara, K.; De Schryver, F.C.; Van der Auweraer, M.; Tobe, Y.; De Feyter, S. Structural transformation of a two-dimensional molecular network in response to selective guest inclusion. Angew. Chem. Int. Ed. 2007, 46, 2831-2834. b) McGonigal, G. C.; Bernhardt, R. H.; Thomson, D. J. Imaging Alkane Layers at the Liquid/graphite Interface with the Scanning Tunneling Microscope. Applied Physics Letters 1990, 57, 28. c) Hentschke, R.; Schürmann, B. L.; Rabe, J. P. Molecular Dynamics Simulations of Ordered Alkane Chains Physisorbed on Graphite. The Journal of Chemical Physics 1992, 96, 6213. d) Marchenko, A.; Xie, Z.-X.; Cousty, J.; Pham Van, L. Structures of SelfAssembled Monolayer of Alkanes Adsorbed on Au(111) Surfaces. Surface and Interface Analysis 2000, 30, 167-169. e) Cyr, D. M.; Venkataraman, B.; Flynn, G. W. STM Investigations of Organic Molecules Physisorbed at the Liquid-Solid Interface. Chemistry of materials 1996, 8, 1600-1615. f) Groszek, A. J. Selective Adsorption at Graphite/Hydrocarbon Interfaces. Proc. R. Soc. London, Ser. A 1970, 314, 473480.

(4) a) El Garah, M.; Marets, N.; Mauro, M.; Aliprandi, A.; Bonacchi, S.; De Cola, L.; Ciesielski, A.; Bulach, V.; Hosseini, M.W.; Samori, P. Nanopatterning of surfaces with monometallic and heterobimetallic 1D coordination polymers : a molecular tectonics approach at the solid/liquid interface. J. Am. Chem. Soc. 2015, 137, 8450-8459. b) El Garah, M.; Ciesielski, A.; Marets, N.; Bulach, V.; Hosseini, M.W.; Samori, P. Molecular tectonics based nanopatterning of interfaces with 2D metal-organic frameworks (MOFs). Chem. Comm. 2014, 50, 12250-12253. c) Eddaoudi, M.; Moler, D.B.; Li, H.; Chen, B.; Reineke, T.M.; O'Keeffe, M.; Yaghi, O.M. Modular chemistry: secondary building units as a basis for the design of highly porous and robust metal-organic carboxylate frameworks. Acc. Chem. Res. 2001, 34, 319-330. d) Kole, G.K.; Vittal, J.J. Solid-state reactivity and structural transformations involving coordination polymers. Chem. Soc. Rev. 2013, 42, 1755-1775. e) Diaz, D. J.; Bernhard, S.; Storrier, G. D.; Abruna, H. D. Redox active ordered arrays via metal initiated self-assembly of terpyridine based ligands. J. Phys. Chem. 2001, B 105, 8746-8754. f) Stepanow, S.; Lingenfelder, M.; Dmitriev, A.; Spillmann, H.; Delvigne, E.; Lin, N.; Deng, X.; Cai, C.; Barth, J.V.; Kern, K.;. Steering molecular organization and host-guest interactions using two-dimensional nanoporous coordination systems. Nature Mater. 2004, 3, 229233. 
(5) a) Arrigoni, C.; Schull, G.; Bléger, D.; Douillard, L.; Fiorini-Debuisschert, C.; Mathevet, F.; Kreher, D.; Attias, A.-J.; Charra, F. Structure and Epitaxial Registry on Graphite of a Series of Nanoporous SelfAssembled Molecular Monolayers. J. Phys. Chem. Lett. 2010, 1 (1), 190-194. b) Szabelski, P.; Rżysko, W.; Pańczyk, T.; Ghijsens, E.; Tahara, K.; Tobe, Y.; Feyter, S. D. Self-Assembly of Molecular Tripods in Two Dimensions: Structure and Thermodynamics from Computer Simulations. RSC Adv. 2013, 3 (47), 25159-25165.

(6) Zhang, H.-M.; Xie, Z.-X.; Mao, B.-W.; Xu, X. Self-Assembly of Normal Alkanes on the Au (111) Surfaces. Chem. - Eur. J. 2004, 10 (6), 1415-1422.

(7) Li, J.; Wieghold, S.; Öner, M. A.; Simon, P.; Hauf, M. V.; Margapoti, E.; Garrido, J. A.; Esch, F.; Palma, C.A.; Barth, J. V. Three-Dimensional Bicomponent Supramolecular Nanoporous Self-Assembly on a Hybrid All-Carbon Atomically Flat and Transparent Platform. Nano Lett. 2014, 14 (8), 4486-4492.

(8) Comstock, M. J.; Levy, N.; Kirakosian, A.; Cho, J.; Lauterwasser, F.; Harvey, J. H.; Strubbe, D. A.; Fréchet, J. M. J.; Trauner, D.; Louie, S. G.; et al. Reversible Photomechanical Switching of Individual Engineered Molecules at a Metallic Surface. Phys. Rev. Lett. 2007, 99 (3), 38301.

(9) Baisch, B.; Raffa, D.; Jung, U.; Magnussen, O. M.; Nicolas, C.; Lacour, J.; Kubitschke, J.; Herges, R. Mounting Freestanding Molecular Functions onto Surfaces: The Platform Approach. J. Am. Chem. Soc. 2009, 131 (2), 442-443.

(10) Bléger, D.; Kreher, D.; Mathevet, F.; Attias, A.-J.; Schull, G.; Huard, A.; Douillard, L.; Fiorini-Debuischert, C.; Charra, F. Surface Noncovalent Bonding for Rational Design of Hierarchical Molecular SelfAssemblies. Angew. Chem. 2007, 119 (39), 7548-7551.

(11) Groszek, A. J. Selective Adsorption at Graphite/Hydrocarbon Interfaces. Proc. R. Soc. Lond. Math. Phys. Eng. Sci. 1970, 314 (1519), 473-498.

(12) Du, P.; Jaouen, M.; Bocheux, A.; Bourgogne, C.; Han, Z.; Bouchiat, V.; Kreher, D.; Mathevet, F.; FioriniDebuisschert, C.; Charra, F.; et al. Surface-Confined Self-Assembled Janus Tectons: A Versatile Platform towards the Noncovalent Functionalization of Graphene. Angew. Chem. 2014, 126 (38), 10224-10230.

(13) Bléger, D.; Bocheux, A.; Kreher, D.; Mathevet, F.; Attias, A.-J.; Metgé, G.; Douillard, L.; FioriniDebuisschert, C.; Charra, F. An Optimized Alkyl Chain-Based Binding Motif for 2D Self-Assembly: A Comprehensive Crystallographic Approach. Nanoscale 2013, 5 (4), 1452-1455.

(14) Clair, S.; Pons, S.; Seitsonen, A. P.; Brune, H.; Kern, K.; Barth, J. V. STM Study of Terephthalic Acid SelfAssembly on $\mathrm{Au}(111)$ : Hydrogen-Bonded Sheets on an Inhomogeneous Substrate. J. Phys. Chem. $B$ 2004, 108 (38), 14585-14590.

(15) Cebula, I.; Smith, E. F.; Gimenez-Lopez, M. del C.; Yang, S.; Schröder, M.; Champness, N. R.; Beton, P. H. Packing of Isophthalate Tetracarboxylic Acids on $\mathrm{Au}(111)$ : Rows and Disordered Herringbone Structures. J. Phys. Chem. C 2013, 117 (36), 18381-18385.

(16) Bléger, D.; Kreher, D.; Mathevet, F.; Attias, A.-J.; Arfaoui, I.; Metgé, G.; Douillard, L.; FioriniDebuisschert, C.; Charra, F. Periodic Positioning of Multilayered [2.2]Paracyclophane-Based Nanopillars. Angew. Chem. Int. Ed. 2008, 47 (44), 8412-8415.

(17) Chang, H.-T.; Lee, H.-T.; Chang, E.-C.; Yeh, M.-Y. Synthesis and Characterization of the Luminescent Poly[2-Decyloxy-5-(2',5'-Bis(decyloxy)phenyl)-1,4-Phenylenevinylene]. Int. J. Polym. Mater. Polym. Biomater. 2007, 56 (5), 483-498.

(18) Moy, C. L.; Kaliappan, R.; McNeil, A. J. Aryl Trihydroxyborate Salts: Thermally Unstable Species with Unusual Gelation Abilities. J. Org. Chem. 2011, 76 (20), 8501-8507.

(19) Moorthy, J. N.; Venkatakrishnan, P.; Mal, P.; Venugopalan, P. Solid-State Diphotocyclization of Iso- and Terephthalaldehydes via Dihalogen Substitution. J. Org. Chem. 2003, 68 (2), 327-330.

(20) Gray, R.; Boekelheide, V. A Study of the Synthesis and Properties of [2.2.2.2](1,2,4,5)cyclophane. J. Am. Chem. Soc. 1979, 101 (8), 2128-2136. 